\title{
Correlation of finite element models of multi-physics systems
}

\author{
K.K. Sairajan *, G.S. Aglietti, Scott J.I. Walker \\ Astronautics Research Group, University of Southampton, Southampton SO17 1BJ, United Kingdom
}

\section{A R T I C L E I N F O}

Article history:

Received 12 September 2013

Received in revised form

31 March 2014

Accepted 3 April 2014

Handling Editor: H. Ouyang

Available online 9 May 2014

\begin{abstract}
A B S T R A C T
The modal assurance criterion (MAC) and normalized cross-orthogonality (NCO) check are widely used to assess the correlation between the experimentally determined modes and the finite element model (FEM) predictions of mechanical systems. Here, their effectiveness in the correlation of FEM of two types of multi-physics systems, namely, viscoelastic damped systems and a shunted piezoelectric system are investigated using the dynamic characteristics obtained from a nominal FEM, that are considered as the 'true' or experimental characteristics and those obtained from the inaccurate FEMs. The usefulness of the MAC and NCO check in the prediction of the overall loss factor of the viscoelastic damped system, which is an important design tool for such systems, is assessed and it is observed that these correlation methods fail to properly predict the damping characteristics, along with the responses under base excitation. Hence, base force assurance criterion (BFAC) is applied by comparing the 'true' dynamic force at the base and inaccurate FEM predicted force such that the criterion can indicate the possible error in the acceleration and loss factor. The effect of temperature as an uncertainty on the MAC and NCO check is also studied using two viscoelastic systems. The usefulness of MAC for the correlation of a second multi-physics FEM that consists of a shunted piezoelectric damped system is also analyzed under harmonic excitation. It has been observed that MAC has limited use in the correlation and hence, a new correlation method - current assurance criterion - based on the electric current is introduced and it is demonstrated that this criterion correlates the dynamic characteristics of the piezoelectric system better than the MAC.
\end{abstract}

\section{Introduction}

Traditionally in the field of spacecraft structures, we deal with purely mechanical systems/models. However, to control the dynamics of the structure using active/passive methods or to enhance the structural performance of aerospace systems, the analysis of multi-physics models becomes necessary. A method to assess the accuracy of such models is vital to improve the analytical predictions and the widespread use of the modeling techniques. Here, two types of widely employed multiphysics systems, namely, viscoelastic damped system and an electric circuit-fed piezoelectric system under base excitation are considered. Base excitation is generally used to qualify a structure for aerospace applications and the results can be used to verify the design margins and to update the FEMs [1]. Although a number of FEM correlation methods such as the MAC [2], NCO check [3], COMAC [4] and FRAC [5] are available for conventional structures, a specific correlation method for multi-physics system has not been reported. Moreover, these subsystems are developed independently and sometimes

\footnotetext{
* Corresponding author. Present address: ISRO Satellite Centre, Vimanapura, Bangalore 560017, India. Tel.: +91 8025083672.

E-mail address: kk_sairaj@yahoo.com (K.K. Sairajan).
} 
supplied by an external organization. A specific correlation method for the subsystems will help to suitably assess the system before it is integrated into the main structure. Here, the correlations of FEMs of viscoelastic and shunted systems are assessed using common and simple configurations. However, the concept can be adapted to more complex systems.

Viscoelastic materials have been effectively used to reduce the vibration response of light weight structures such as spacecraft, reaction wheel assemblies, and airplane fuselages [6]. These materials can also be used to increase the damping in plates and sandwich structure and this passive damping method is easy to implement and more economical than active damping techniques [7]. For example, in spacecraft structure, thin aluminium plates embedded with viscoelastic materials can be used to mount small antennae such as telemetry and tele-command patch antennae or micro strip antennae. Honeycomb sandwich panels have widespread use in aerospace structures to form equipment mounting decks and solar panels. These panels are generally larger in dimension than the thin plates mentioned previously along with higher load bearing capability. The capability of viscoelastic material to exhibit properties of both a viscous fluid and an elastic solid is effectively used to absorb the vibration energy and thereby reduce the structural responses. The amount of viscous or elastic behavior depends primarily on the temperature of the system and the frequency or the rate of loading. The effectiveness of the damping of such systems is generally assessed using the modal loss factor of a specified mode of interest. A modal loss factor is the ratio of the total energy dissipated per cycle to the maximum amount of energy stored during the cycle [8].

The accurate determination of the loss factor is an important step in the design of damped structural systems using viscoelastic material. The pioneering work to determine the loss factor of assemblies consisting of viscoelastic elements using an energy method was performed by Ungar and Kerwin [9]. FEMs have been effectively utilized to analyze the viscoelastic damping in practical systems by many researchers [6,10-12]. Among these methods, the modal strain energy (MSE) [11] method is widely used to determine the loss factor of constrained viscoelastic systems using a commercial finite element program. Here, the MSE method is used to determine the loss factor of two viscoelastic damped systems, namely, a simply supported sandwich plate and a honeycomb sandwich panel constrained at the hold down locations. The first model, Configuration A, simulates a small antenna support structure whereas the second model, Configuration B, simulates the solar panel of a spacecraft along with the hold down locations that are used to assemble the panel to the spacecraft during launch.

The second type of subsystem considered in this study consists of a piezoelectric patch connected with an electric shunt circuit and then bonded to the host structure for vibration damping [13-15]. This system is identified as Configuration C. The relevance of these assemblies is that they are mainly used to control the dynamics of the system in which they are embedded. The electro-elastic systems have been analyzed by different researchers [16-18] and Tzou and Ye [19] used the variational principle to define the piezo-thermoelastic finite elements. The FEM which consists of structural and nonstructural dofs (coupled FEM) has been effectively used for the analysis of piezoelectric systems [20-23] and a commercially available software such as ANSYS [24] provides a convenient way to model more complex and practical systems.

The MAC and NCO check are the most commonly employed correlation tools for validating the conventional structural FEMs $[25,26]$. In this work, the usefulness of these standard methods to correlate the FEMs of the viscoelastic systems is assessed by studying the relationship between the analytical prediction of modal loss factor and these correlation indices. Two different systems are analyzed to consider the various modeling inaccuracies such as boundary conditions, material properties, and hinge stiffness (in the honeycomb sandwich panel). The characteristics of the system determined from the original/nominal FEMs are considered as the experimental or 'true' parameters and those obtained by FEMs with modeling inaccuracies are considered as analytical parameters. The effect of damping on the MAC is analyzed using the complex modes of the system. The recently introduced base force assurance criterion (BFAC) [27] is applied to correlate the viscoelastic systems. Also the effect of temperature on the MAC and NCO is studied using the viscoelastic systems. In addition, the usefulness of the MAC for the correlation of a FEM of a piezoelectric system connected with a shunt electric circuit is analyzed whilst it is subjected to harmonic excitation. A new correlation method called the current assurance criterion (CAC) is defined using the frequency-dependent current in the electric circuit to correlate the shunted piezoelectric system.

\section{Theoretical background}

\subsection{Determination of the loss factor for structural system}

The modal loss factor for the constrained layer damping system can be calculated using the undamped modes and the material loss factor for each constituent material [11]. In the constrained layer damped system, viscoelastic layers are placed between the stiff face sheets. Generally, the face sheet material will have an exceedingly low loss factor compared with the viscoelastic core. Hence, the modal loss factor $\eta^{r}$ for each mode $r$ is approximated as [11]

$$
\eta^{r}=\eta_{v}\left(\frac{V_{v}^{r}}{V_{T}^{r}}\right)
$$

where $\eta_{v}$ denotes the loss factor of the core at the resonance frequency of mode $r, V_{v}^{r}$ and $V_{T}^{r}$ are the elastic strain energy of the viscoelastic material alone and the strain energy of the total system during the mode $r$. Generally, the ratio, $\eta^{r} / \eta_{v}$, can be directly obtained from the FEM results as the ratio of the modal strain energy of the core to the total elastic strain energy. In this study, $3 \mathrm{M}$ viscoelastic materials are considered for the damping layers and $\eta_{v}$ is taken as 1.0 at $30{ }^{\circ} \mathrm{C}$ for the core in the desired frequency range [28]. Although this modal strain energy method computes the loss factor from the undamped 
normal modes, it gives a reasonably good approximation of the damping and leads to an easy way of designing the damping system. However, the frequency-dependent material properties of the core need some approximation to accommodate this method. The derivation of Eq. (1) is shown in Appendix A.

\subsection{Electro-elastic system}

Piezoelectric materials are used in structural vibration control and in transducer technology. A closed form solution to find the coupled effect of the electrical and structural behavior is limited to a simple configuration as the equations of piezoelectricity are quite complex. Hence, Allik and Hughes [17] proposed a general method for electro-elastic analysis by incorporating the piezoelectric effect in the finite element formulation as described below. For a linear material behavior, the constitutive equations for a piezoelectric crystal can be written as

$$
\begin{gathered}
\boldsymbol{\sigma}=C \boldsymbol{\varepsilon}-\mathbf{P e} \\
\mathbf{d}=\mathbf{P}^{T} \boldsymbol{\varepsilon}+\mathbf{D e}
\end{gathered}
$$

where $\boldsymbol{\sigma}$ denotes the stress tensor, $C$ is the elastic stiffness tensor evaluated at a constant electric field, $\varepsilon$ is mechanical strain, $\mathbf{P}$ is the piezoelectric tensor, $\mathbf{e}$ is the electric field, $\mathbf{d}$ is the electric displacement vector, and $\mathbf{D}$ is the dielectric tensor evaluated at constant mechanical strain. Then using the variational principle for the electro-mechanical system, two equilibrium equations for nodal displacement, $\mathbf{x}_{i}$, and electric potential, $\mathbf{v}_{i}$, can be written as

$$
\begin{gathered}
\mathbf{M} \ddot{\mathbf{x}}_{i}+\mathbf{K}_{x x} \mathbf{x}_{i}+\mathbf{K}_{x v} \mathbf{v}_{i}=\mathbf{f}_{B}+\mathbf{f}_{S}+\mathbf{f}_{p} \\
\mathbf{K}_{v x} \mathbf{x}_{i}+\mathbf{K}_{v v} \mathbf{v}_{i}=\mathbf{q}_{B}+\mathbf{q}_{S}+\mathbf{q}_{p} .
\end{gathered}
$$

Here, $\ddot{\mathbf{x}}$ represents the acceleration, and the details of other individual terms in the equilibrium equations are given in Appendix B. The system level equation can be formed by the nodal addition of elemental contributions. The assembled equation for the complete system with damping can be written in the matrix form as

$$
\left[\begin{array}{cc}
\mathbf{M} & \mathbf{0} \\
\mathbf{0} & \mathbf{0}
\end{array}\right]\left\{\begin{array}{l}
\ddot{\mathbf{x}} \\
\ddot{\mathbf{v}}
\end{array}\right\}+\left[\begin{array}{cc}
\mathbf{C}_{x x} & \mathbf{0} \\
\mathbf{0} & \mathbf{0}
\end{array}\right]\left\{\begin{array}{c}
\dot{\mathbf{x}} \\
\dot{\mathbf{v}}
\end{array}\right\}+\left[\begin{array}{ll}
\mathbf{K}_{x x} & \mathbf{K}_{x v} \\
\mathbf{K}_{v x} & \mathbf{K}_{v v}
\end{array}\right]\left\{\begin{array}{l}
\mathbf{x} \\
\mathbf{v}
\end{array}\right\}=\left\{\begin{array}{c}
\mathbf{f}_{T} \\
\mathbf{q}_{T}
\end{array}\right\}
$$

where $\mathbf{C}_{x x}$ is the mechanical damping matrix, $\mathbf{f}_{T}$ and $\mathbf{q}_{T}$ are the total applied force and charge, respectively. It should be noted that all the nodes on one electrode surface of the piezoelectric patch will have identical potential. This indicates that there is a single potential degree of freedom (dof) per electrode of piezoelectric patch.

\subsubsection{Free vibration analysis}

A piezoelectric patch can be configured either in an open circuit or in a closed circuit form. The open circuit configuration is also known as the sensor configuration and the potential difference between the electrodes depends on the mechanical load acting on the system. Let the harmonic displacement and electric potential be given by [13]

$$
\begin{aligned}
& \mathbf{x}=\mathbf{x}_{0} \mathrm{e}^{\mathrm{i} \omega t} \\
& \mathbf{v}=\mathbf{v}_{0} \mathrm{e}^{\mathrm{i} \omega t}
\end{aligned}
$$

where $\omega$ is the circular frequency and $t$ represents the time. For harmonic motion, Eq. (6) can be re-written for the undamped free vibration analysis as

$$
\left[\begin{array}{cc}
\mathbf{K}_{x x}-\omega^{2} \mathbf{M} & \mathbf{K}_{x v} \\
\mathbf{K}_{v x} & \mathbf{K}_{v v}
\end{array}\right]\left\{\begin{array}{l}
\mathbf{x}_{0} \\
\mathbf{v}_{0}
\end{array}\right\} \mathrm{e}^{\mathrm{i} \omega t}=\mathbf{0} .
$$

The lower part of this equation gives

$$
\mathbf{v}=-\mathbf{K}_{v v}^{-1} \mathbf{K}_{v x} \mathbf{x}
$$

Substituting the value of electric potential into the upper part of Eq. (9) yields

$$
\left(\left(\mathbf{K}_{x x}-\mathbf{K}_{x v} \mathbf{K}_{v v}^{-1} \mathbf{K}_{v x}\right)-\boldsymbol{\omega}_{o}^{2} \mathbf{M}\right) \phi_{o}=\mathbf{0} .
$$

This is the equation of the generalized eigenvalue problem. Here, $\omega_{o}$ is the modal frequency of the open circuit configuration and $\boldsymbol{\phi}_{o}$ is the corresponding mode shape. In the case of a short circuit configuration, $\mathbf{v}_{o}$ is zero, hence the eigenvalue equation reduces to

$$
\left(\mathbf{K}_{x x}-\omega_{s}^{2} \mathbf{M}\right) \phi_{s}=\mathbf{0}
$$

where $\omega_{s}$ is the modal frequency of the closed circuit configuration and $\phi_{s}$ is the corresponding mode shape. 


\subsubsection{Response analysis of the piezoelectric structure under harmonic excitation}

The response of the system under harmonic excitation can be computed by solving the time-dependent equation of motion given in Eq. (6). Due to the presence of damping, there will be a phase shift. Hence, the displacement and electric field can be expressed as [14]

$$
\begin{aligned}
& \mathbf{x}(\boldsymbol{t})=\mathbf{x e}^{-\mathrm{i} \Omega t+\varphi_{1}} \\
& \mathbf{v}(\boldsymbol{t})=\mathbf{v} e^{-\mathrm{i} \Omega t+\varphi_{2}}
\end{aligned}
$$

where $\Omega$ represents the forcing frequency and $\varphi_{1}$ and $\varphi_{2}$ denote the phase shift. Similarly, the applied force and charge are represented by

$$
\begin{aligned}
\mathbf{f}_{T}(\boldsymbol{t}) & =\mathbf{f}_{T} \mathrm{e}^{-\mathrm{i} \Omega t+\varphi_{3}} \\
\mathbf{q}_{T}(\boldsymbol{t}) & =\mathbf{q}_{T} \mathrm{e}^{-\mathrm{i} \Omega t+\varphi_{4}} .
\end{aligned}
$$

where $\varphi_{3}$ and $\varphi_{4}$ denote the phase shift. Incorporating Eqs. (13)-(16) into Eq. (6) gives

$$
\left[\begin{array}{cc}
\mathbf{K}_{x x}-i \Omega \mathbf{C}_{x x}-\Omega^{2} \mathbf{M} & \mathbf{K}_{x v} \\
\mathbf{K}_{v x} & \mathbf{K}_{v v}
\end{array}\right]\left\{\begin{array}{l}
\mathbf{x} \\
\mathbf{v}
\end{array}\right\}=\left\{\begin{array}{l}
\mathbf{f}_{T} \\
\mathbf{q}_{T}
\end{array}\right\} .
$$

It can be noted that when the natural frequency of the structure hosting the piezoelectric system coincides with the forcing frequency, this leads to the peak harmonic response.

\subsubsection{Analysis of the piezoelectric structure connected with an electric shunt circuit}

To have compatibility between the piezoelectric formulation and the standard electric circuit equation, the latter generally follows Kirchoff's current law; the electric charge balance at each node is enforced [20]. Thus, the circuit elements, namely, capacitor $(C)$, resistor $(R)$ and inductor $(L)$ were represented by an equivalent capacitance matrix form. For a harmonic analysis, the capacitor is represented by the equation [14,29]:

$$
C\left[\begin{array}{cc}
1 & -1 \\
-1 & 1
\end{array}\right]\left\{\begin{array}{l}
v_{1} \\
v_{2}
\end{array}\right\}=\left\{\begin{array}{l}
0 \\
0
\end{array}\right\} .
$$

A resistor is expressed for a harmonic analysis as

$$
\mathrm{i} \Omega\left(-\frac{1}{\Omega^{2} R}\right)\left[\begin{array}{cc}
1 & -1 \\
-1 & 1
\end{array}\right]\left\{\begin{array}{l}
v_{1} \\
v_{2}
\end{array}\right\}=\left\{\begin{array}{l}
0 \\
0
\end{array}\right\}
$$

and an inductor by

$$
\left(-\frac{1}{\Omega^{2} L}\right)\left[\begin{array}{cc}
1 & -1 \\
-1 & 1
\end{array}\right]\left\{\begin{array}{l}
v_{1} \\
v_{2}
\end{array}\right\}=\left\{\begin{array}{l}
0 \\
0
\end{array}\right\}
$$

where $v_{1}$ and $v_{2}$ are the electric potential dofs at the nodes where these circuit elements are connected. It is worth mentioning that these equations can be readily added to Eq. (6) to couple the electric circuit effect to the piezoelectric structure.

\section{Configuration and modeling of multi-physics systems}

\subsection{Viscoelastic systems}

To study the correlation of FEMs containing viscoelastic materials, two systems - Configuration A and Configuration B have been modeled in Nastran [30]. Configuration A consists of a sandwich plate with a length of $0.3 \mathrm{~m}$ and width of $0.2 \mathrm{~m}$. The FEM and the cross sectional details in millimeters $(\mathrm{mm})$ are shown in Fig. 1 and this represents a small antenna support structure of a spacecraft. The second system (Configuration B) represents a typical solar panel of a spacecraft. The FEM, the axis system and the cross sectional details are given in Fig. 2. In Configuration A, the viscoelastic core is constrained between the two identical aluminium face sheets and this has been modeled using a single layer of eight node hexagonal elements (HEX), whereas the face sheets were modeled using four nodes quadrilateral shell elements (QUAD4) of Nastran [11]. The properties of different materials used in the FEM are given in Table $1[11,28]$. For the viscoelastic material, the 3M viscoelastic polymer properties at $30^{\circ} \mathrm{C}$ and $50 \mathrm{~Hz}$ have been considered for the analysis of the sandwich plate. The frequency at which the material properties were taken is the average frequency of the two target modes of the system (described in Section 4.1). As the viscoelastic properties at all frequencies are not available, estimated values using Ref. [28] are shown in Table 1.

A simply supported boundary condition along the edges of the entire system has been applied and these constrained dofs were rigidly connected together to a single node located at the base $[0.15,0.1,-0.02]$. This base node is located directly below the diagonal intersection of the bottom face sheet as the nodes on the lower face sheet are located at $z=0$ plane. The 
(a)

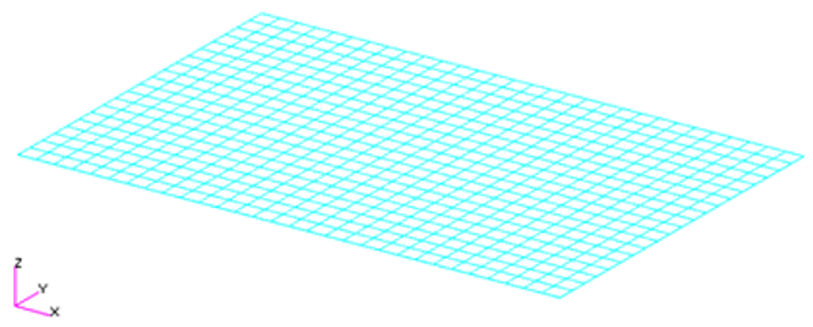

(b)

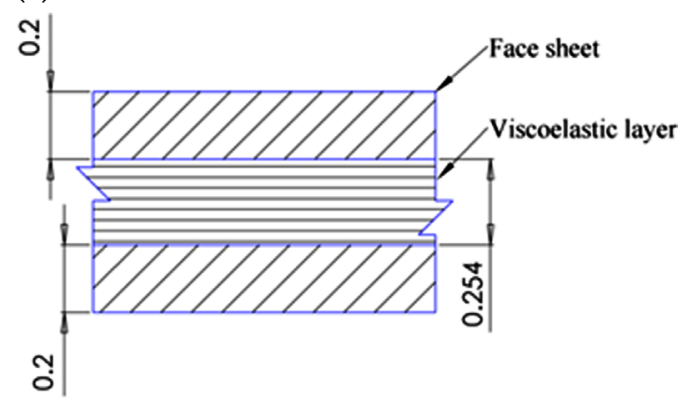

Fig. 1. Model of a constrained layer viscoelastic system - Configuration A: (a) FEM and the axis system and (b) cross sectional view (all dimensions are in $\mathrm{mm}$ ).

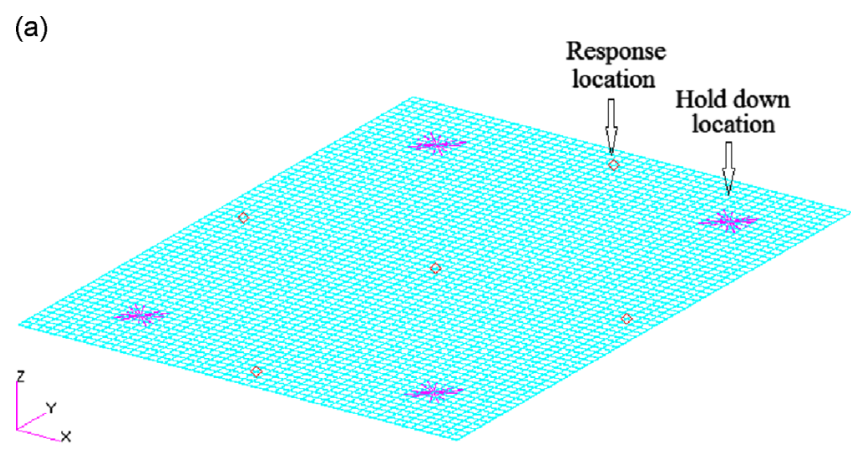

(b)

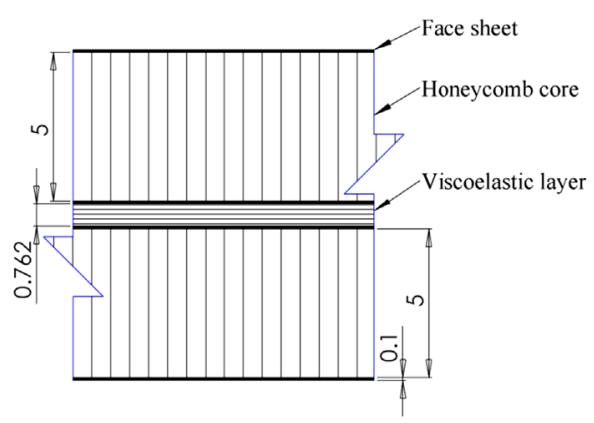

Fig. 2. Details of a honeycomb sandwich panel model - Configuration B: (a) FEM and axis system and (b) cross sectional view (all dimensions are in mm).

Table 1

Material properties of viscoelastic system.

\begin{tabular}{lllll}
\hline Property & Material & & \\
\cline { 2 - 4 } & Honeycomb core & Aluminium & $3 \mathrm{M}$ & \\
\cline { 3 - 4 } & & & $50(\mathrm{~Hz})$ & $132(\mathrm{~Hz})$ \\
\hline Young's modulus (MPa) & $E_{11}=1.0 \times 10^{-3}$ & $E=68.9 \times 10^{3}$ & & $G=3.6$ \\
Shear modulus (MPa) & $E_{22}=1.0 \times 10^{-3}$ & & $G=2.0$ & 0.49 \\
Poisson's ratio, $\nu$ & $G_{13}=138.0$ & & 0.49 & 999.0 \\
Density, $\rho\left(\mathrm{kg} / \mathrm{m}^{3}\right)$ & $G_{23}=138.0$ & 0.3 & 999.0 & 1.0 \\
Material loss factor $\left(\right.$ at $\left.30{ }^{\circ} \mathrm{C}\right)$ & 32.0 & 2740.0 & 1.0 & \\
\hline
\end{tabular}

Subscripts 1 and 2 indicate the in-plane directions; 3 thickness direction. 
base excitation is applied to this node in the subsequent analysis. The total mass of the system is $80.98 \times 10^{-3} \mathrm{~kg}$ and the coordinate system of the FEM is shown in Fig. 1 a.

In Configuration $\mathrm{B}$, the chosen solar panel has a rectangular shape $(0.8 \mathrm{~m} \times 0.6 \mathrm{~m})$ and the viscoelastic layers are constrained between the two identical sandwich panels and each of these sandwich panels consists of two thin aluminium face sheets $\left(1.0 \times 10^{-4} \mathrm{~m}\right.$ thick $)$ and a honeycomb core as shown in Fig. $2 \mathrm{~b}$. This configuration is chosen to obtain the maximum damping effect by placing the viscoelastic layers near the maximum shear stress area along with the manufacturing feasibility of the sandwich assembly. The honeycomb sandwich panels are modeled using layered shell elements (PSHELL) of Nastran and viscoelastic layers are modeled using eight nodes hexagonal elements. The solar panel model consists of four identical hold down blocks, which support the panel to the main spacecraft during the launch. Each hold down has an axial stiffness of $1.0 \times 10^{10} \mathrm{~N} / \mathrm{m}$ and a rotational stiffness of $1.0 \times 10^{8} \mathrm{~N} / \mathrm{rad}$. The nodes in each hold down area were rigidly connected together and connected to the base point using six CELAS elements, the first three elements correspond to the three axial stiffness and the last three correspond to the rotational stiffness. A single base fixed boundary condition is obtained by rigidly connecting the four base points of the hold down blocks at the bottom of the panel.

The material properties used to model Configuration B are also given in Table 1. Here, the properties of the $3 \mathrm{M}$ viscoelastic damping polymer at $30{ }^{\circ} \mathrm{C}$ and $132 \mathrm{~Hz}$, that have been estimated using Ref. [28], were used in the analysis. It should be noted that $132 \mathrm{~Hz}$ is the average frequency of the target modes of the system as described in Section 4.1 . It was also considered that the structure supports an additional mass of $2.0 \mathrm{~kg}$, which accounts for solar cells, harness etc. and this non-structural mass is uniformly distributed over the top surface of the panel. The total mass of the system was $3.04 \mathrm{~kg}$.

\subsection{Piezoelectric system}

To study the correlation aspects of a piezoelectric system, a piezoelectric patch bonded onto the upper face of a titanium plate was considered and the geometry of the configuration is shown in Fig. 3. Both the plate and piezoelectric patch have a rectangular shape with a uniform thickness of $0.002 \mathrm{~m}$ and $3.8 \times 10^{-4} \mathrm{~m}$, respectively. A resistor and inductor are connected in series between the top and the bottom electrodes of the piezoelectric patch to dissipate the vibration energy of the system. A similar problem has been analyzed by Min et al. [14] but here the focus is to study the correlation aspects of the multi-physics system rather than the vibration control.

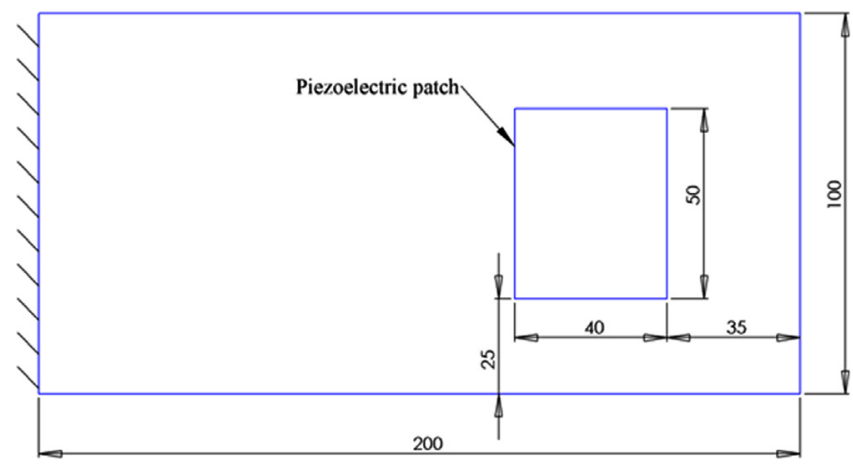

(All dimensions are in $\mathrm{mm}$ )

Fig. 3. Details of the piezoelectric patch on the titanium plate - Configuration $C$ (all dimensions are in mm)

The FEM of the piezoelectric system attached with a resistor $(R)$ and inductor $(L)$ in series is shown in Fig. 4. The piezoelectric patch is placed in such a way that it can reduce the dynamic response due to the third bending mode of the structure when it is excited by a harmonic force $\left(\mathbf{f}_{T}\right)$ at the fixed end in the $Z$ direction as shown in Fig. 4 . The modeling and the analysis were conducted using ANSYS multi-physics software. The titanium plate was modeled using 20-node SOLID186 elements and the element has three displacement degrees of freedom per node [31]. The piezoelectric patch was modeled using SOLID226 elements with the material property of PZT-5 and the resistor and inductor were modeled using CIRCU94 elements. These elements are capable of simulating the linear electric circuits and can be directly coupled with the piezoelectric elements [32]. The properties of titanium and PZT-5 used in the analysis are shown in Table 2 [14]. The poling direction for the piezoelectric material is chosen as the thickness direction of the patch. The bonding details of the piezoelectric patch to the plate are not considered in this analysis. 


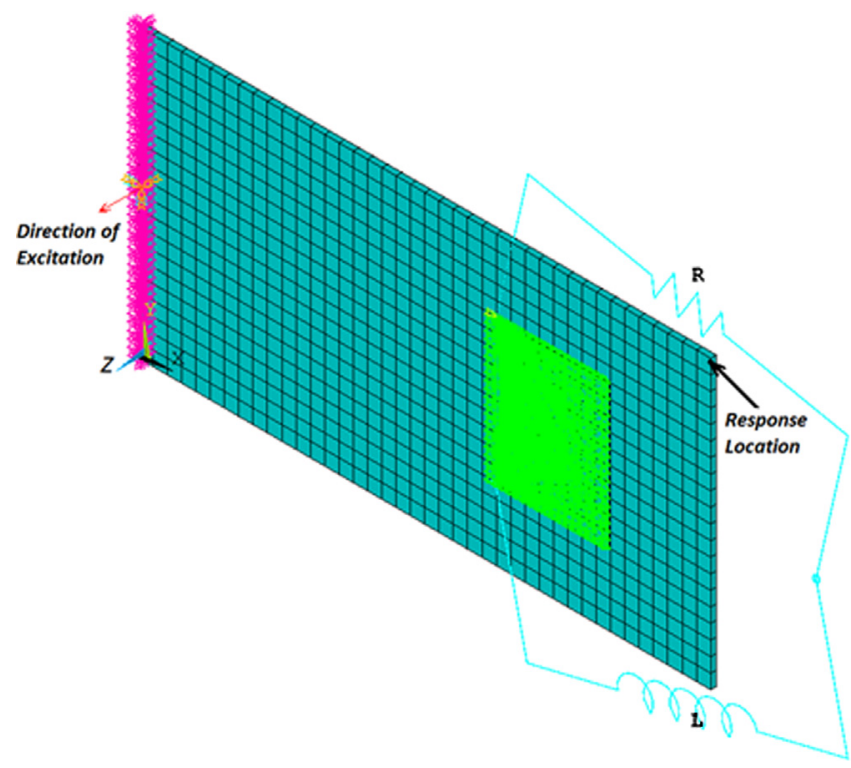

Fig. 4. FEM of the piezoelectric system.

Table 2

Material properties of piezoelectric system.

\begin{tabular}{lll}
\hline \multirow{2}{*}{ Property } & Material & \\
\cline { 2 - 3 } & PZT-5 & Titanium \\
\hline Young's modulus (GPa) & $E_{11}=E_{22}=E_{33}=77$ & $E=104.83$ \\
Poisson's ratio & $\nu_{12}=\nu_{23}=\nu_{13}=0.3$ & $\nu=0.3$ \\
Density $\left(\mathrm{kg} / \mathrm{m}^{3}\right)$ & $\rho=7700$ & $\rho=4428.83$ \\
Shear modulus $(\mathrm{GPa})$ & $G_{12}=G_{23}=G_{13}=29.62$ & \\
Piezoelectric coefficients $\left(10^{-12} \mathrm{~m} / \mathrm{V}\right)$ & $d_{31}=d_{32}=-130$ & \\
& $d_{33}=330$ & \\
Electric permittivity & $d_{24}=d_{15}=327$ & \\
\hline$\epsilon_{0}=8.85 \times 10^{-12} \mathrm{~F} / \mathrm{m}$; electric permittivity of vacuum. & $\epsilon_{11} / \epsilon_{0}=\epsilon_{22} / \epsilon_{0}=\epsilon_{33} / \epsilon_{0}=1300$ & \\
\end{tabular}

\section{Results and discussions}

\subsection{Viscoelastic systems}

An undamped free vibration analysis of the two viscoelastic systems shown in Figs. 1 and 2 was carried out and the modal loss factors of the important modes were determined. The loss factor of each mode is determined as the ratio of strain energy of the core to the total strain energy of the corresponding mode [11]. The obtained results for both the systems are given in Table 3. In the case of the sandwich plate, the effective mass of modes 2, 3 and 5 in translation were negligible (less than $10^{-11} \mathrm{~kg}$ ), hence the first and the fourth modes only are considered as the target modes for the correlation study, which have 2 percent or more effective mass [33]. However, the first and fifth modes of the solar panel model have more than 2 percent effective mass and hence these modes were taken as the target modes for the system. These target modes have an effective mass of $1.63 \mathrm{~kg}$ and $0.55 \mathrm{~kg}$, respectively, in the $Z$ direction. The results obtained from theses nominal FEMs were considered as the experimental or 'true' characteristics in the correlation.

To perform the FEM correlation of the viscoelastic systems, twelve intentionally erroneous FEMs were created from the nominal FEMs of the aluminium sandwich plate and the sandwich solar panel by changing the boundary conditions of the structure. Some erroneous models were created by changing both the boundary conditions and the material properties. In the case of solar panel FEM, the hold down stiffness was also varied to generate different FEMs. These errors are introduced to simulate the inaccuracies and possible modeling errors present in the FEM. However, the cross sectional symmetry is maintained in all the models but not the boundary condition symmetry. The target modes of different models and the nominal model of the sandwich plate were determined using the 28 nodes in the FEM, which represent the 28 accelerometers in the $Z$ direction (Fig. 1a). The sensor locations are spread across the upper face sheet of the sandwich plate. The modal frequencies and the corresponding loss factors of the target modes of these erroneous models are given in Table 4. The two target modes of 
Table 3

Dynamic characteristics of the nominal FEMs.

\begin{tabular}{lcll}
\hline Mode number & Frequency $(\mathrm{Hz})$ & Loss factor & Structure \\
\hline 1 & 29.13 & 0.1496 & \\
2 & 44.53 & 0.1368 & Sandwich plate \\
3 & 68.28 & 0.1671 & \\
4 & 70.21 & 0.1271 & \\
5 & 82.32 & 0.1379 & Honeycomb sandwich panel \\
\hline 1 & 72.22 & 0.0887 & \\
2 & 93.44 & 0.0884 & \\
3 & 131.53 & 0.0782 & 0.1229 \\
5 & 164.13 & 0.1154 & \\
\hline
\end{tabular}

Table 4

Dynamic characteristics of different FEMs of Configuration A.

\begin{tabular}{|c|c|c|c|c|}
\hline \multirow[t]{2}{*}{ Model number } & \multicolumn{2}{|l|}{ Target mode 1} & \multicolumn{2}{|l|}{ Target mode 2} \\
\hline & Frequency $(\mathrm{Hz})$ & Loss factor & Frequency $(\mathrm{Hz})$ & Loss factor \\
\hline 1 & 27.56 & 0.1354 & 68.52 & 0.1203 \\
\hline 2 & 26.91 & 0.1514 & 56.82 & 0.1024 \\
\hline 3 & 26.37 & 0.1510 & 66.96 & 0.0966 \\
\hline 4 & 26.53 & 0.1233 & 67.30 & 0.1102 \\
\hline 5 & 25.44 & 0.1118 & 65.11 & 0.0904 \\
\hline 6 & 22.12 & 0.0989 & 59.16 & 0.0848 \\
\hline 7 & 23.81 & 0.0904 & 62.55 & 0.0738 \\
\hline 8 & 22.18 & 0.0826 & 54.03 & 0.0700 \\
\hline 9 & 22.70 & 0.0824 & 61.14 & 0.0683 \\
\hline 10 & 19.11 & 0.0890 & 47.17 & 0.0583 \\
\hline 11 & 19.94 & 0.0729 & 57.36 & 0.0629 \\
\hline 12 & 17.81 & 0.0677 & 46.35 & 0.0606 \\
\hline
\end{tabular}

the honeycomb sandwich panel models (Configuration B) were also determined using the 28 dofs in the $Z$ direction. One of the nominal and the erroneous target mode- 2 of the sandwich panel is shown in Fig. 5. The modal frequencies of the target modes along with the corresponding loss factors for all the different FEMs are given in Table 5.

\subsubsection{Standard correlation methods and the modal loss factor}

The MAC and NCO check were then performed for the target modes using the equations [2]:

$$
\begin{gathered}
\mathbf{M A C}_{l m}=\frac{\left|\boldsymbol{\Psi}_{l}^{\mathrm{T}} \boldsymbol{\phi}_{m}\right|^{2}}{\left(\boldsymbol{\Psi}_{l}^{\mathrm{T}} \boldsymbol{\Psi}_{l}\right)\left(\boldsymbol{\phi}_{m}^{\mathrm{T}} \boldsymbol{\phi}_{m}\right)} \\
\mathbf{N C O}_{l m}=\frac{\left|\boldsymbol{\Psi}_{l}^{\mathrm{T}} \mathbf{M}_{\mathrm{SEREP}} \boldsymbol{\phi}_{m}\right|^{2}}{\left(\boldsymbol{\Psi}_{l}^{\mathrm{T}} \mathbf{M}_{\mathrm{SEREP}} \boldsymbol{\Psi}_{l}\right)\left(\boldsymbol{\phi}_{m}^{\mathrm{T}} \mathbf{M}_{\mathrm{SEREP}} \boldsymbol{\phi}_{m}\right)}
\end{gathered}
$$

where $\boldsymbol{\psi}$ and $\boldsymbol{\phi}$ are the nominal and erroneous target mode shapes, respectively, $\mathbf{M}_{\mathrm{SEREP}}$ is the reduced mass matrix using system equivalent reduction expansion process (SEREP) [32], superscript T represents the transpose and the subscripts $l$ and $m$ vary from one to the number of target modes. The SEREP mass matrix was computed using the normalized modal matrix, $\Psi$, as

$$
\mathbf{M}_{\text {SEREP }}=\left(\boldsymbol{\Psi}^{\dagger}\right)^{\mathrm{T}} \boldsymbol{\Psi}^{\dagger}
$$

where $\boldsymbol{\Psi}^{\dagger}$ is the generalized inverse [34] of the modal matrix $\boldsymbol{\Psi}$. In this study, only the target modes were used in the SEREP reduction [35]. The viscoelastic system considered in this study has more damping than conventional structures such as spacecraft. Therefore to understand the effect of damping on the MAC, the complex modes of the sandwich plate and the honeycomb sandwich panel were computed using Nastran (SOL 107). For the analysis, the average modal loss factor of the target modes of the respective nominal models was used and the obtained mode shapes were considered as 'true' modes. Similarly, the complex modes of the different FEMs were also calculated using the respective average loss factor of the erroneous FEMs of the two systems. The calculated diagonal value of MAC for the two target modes using the complex modes and the real modes for these systems are given in Tables 6 and 7. Here, MAC 1 and MAC 2 correspond to the target mode 1 and target mode 2, respectively. From these results, it can be seen that there is no significant change in the MAC values when the target modes are computed using the real or the complex modes. This indicates that the damping did not 


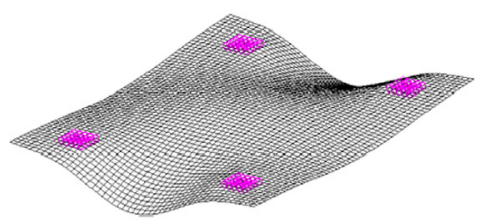

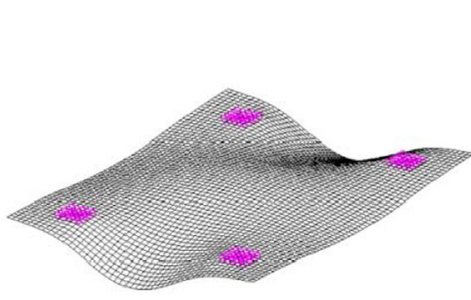

1

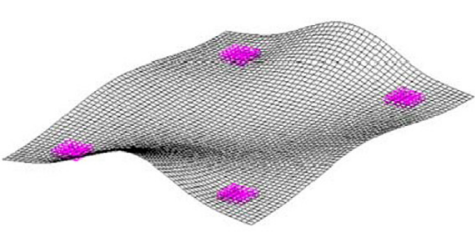

4

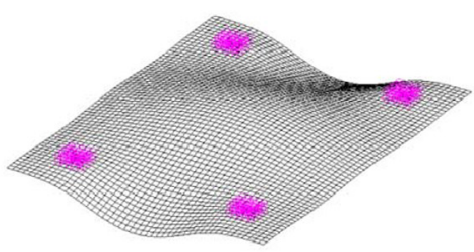

7

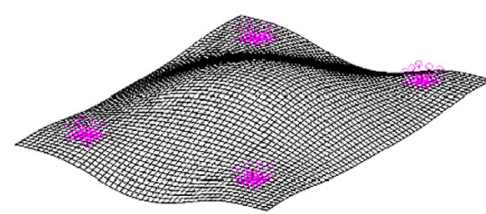

10

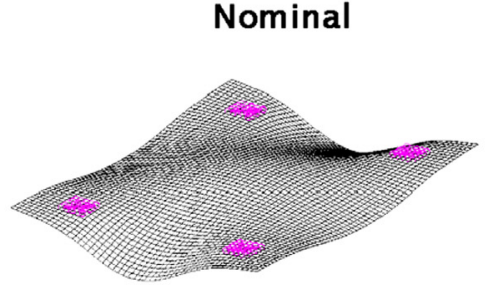

2

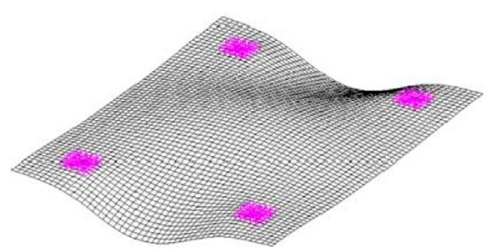

5

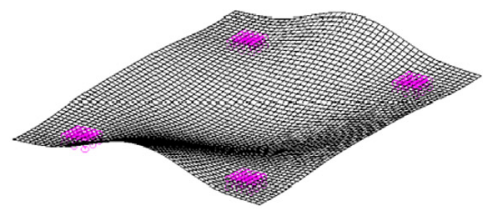

8

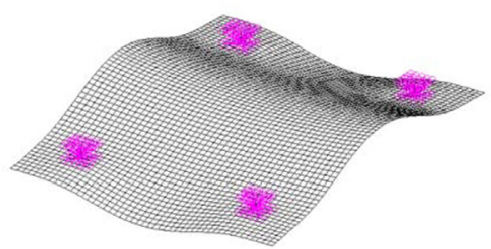

11

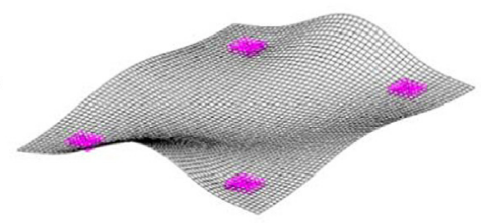

3

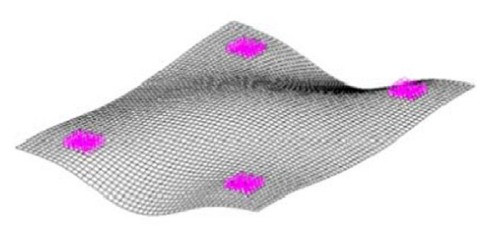

6

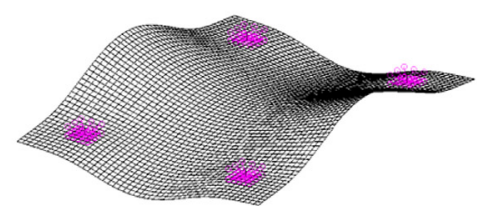

9

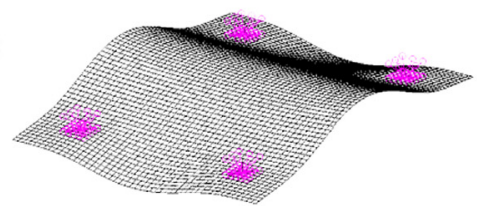

12

Fig. 5. The second target mode of different FEMs of Configuration B.

alter the modes in the considered systems. It should also be noted that the loss factor for all the models considered was less than 15 percent in both the systems and hence real modes are sufficient to calculate the MAC for these types of systems. Therefore only real modes are used for further study.

It is understood that the actual damping in the system is very difficult to model and is usually determined by experimental methods. Here, the damping due to the viscoelastic material alone is considered and attention is limited to its variation with the commonly employed correlation tools MAC and NCO. Although these correlation tools are not suitable for the force response characteristics, they are extensively used for structural model validation. In the remaining section, the variation of the MAC and NCO check with the modal loss factor, as the loss factor is the important design factor in the viscoelastic system, is examined. The percentage error in loss factor, Error ${ }_{\mathrm{LS}}$ in comparison with the corresponding nominal value has been determined for both the target modes using the equation:

$$
\text { Error }_{\mathrm{LS}}=\left|\frac{\mathrm{L} \mathrm{S}_{\mathrm{Nom}}-\mathrm{LS}}{\mathrm{L} \mathrm{S}_{\mathrm{Nom}}}\right| 100
$$


Table 5

Dynamic characteristics of different FEMs of Configuration B.

\begin{tabular}{rllll}
\hline \multirow{2}{*}{ Model number } & \multicolumn{2}{l}{ Target mode 1} & \multicolumn{2}{l}{ Target mode 2} \\
\cline { 2 - 3 } & Frequency (Hz) & Loss factor & & Frequency (Hz) \\
\hline 1 & 72.22 & 0.0887 & 191.04 & 0.1154 \\
2 & 71.92 & 0.0880 & 189.13 & 0.1128 \\
3 & 67.36 & 0.0802 & 180.33 & 0.1041 \\
4 & 67.86 & 0.0764 & 182.65 & 0.1007 \\
5 & 67.64 & 0.0759 & 181.30 & 0.0991 \\
6 & 68.75 & 0.0803 & 171.00 & 0.0855 \\
7 & 65.38 & 0.0711 & 166.98 & 0.0796 \\
9 & 66.10 & 0.0737 & 155.41 & 0.0556 \\
10 & 62.86 & 0.0653 & 152.74 & 0.0614 \\
11 & 55.73 & 0.0519 & 119.66 & 0.0648 \\
& 59.62 & 0.0577 & 139.96 & 0.0517 \\
\end{tabular}

Table 6

Comparison of MAC of Configuration A using real and complex modes.

\begin{tabular}{|c|c|c|c|c|}
\hline \multirow[t]{2}{*}{ Model number } & \multicolumn{2}{|l|}{ MAC 1} & \multicolumn{2}{|l|}{ MAC 2} \\
\hline & Real modes & Complex modes & Real modes & Complex modes \\
\hline 1 & 0.9914 & 0.9914 & 0.9701 & 0.9698 \\
\hline 2 & 0.9349 & 0.9348 & 0.3706 & 0.3704 \\
\hline 3 & 0.8867 & 0.8866 & 0.3607 & 0.3604 \\
\hline 4 & 0.9878 & 0.9878 & 0.9827 & 0.9826 \\
\hline 5 & 0.9853 & 0.9852 & 0.9727 & 0.9725 \\
\hline 6 & 0.9591 & 0.9590 & 0.4494 & 0.4501 \\
\hline 7 & 0.9933 & 0.9932 & 0.9819 & 0.9818 \\
\hline 8 & 0.9762 & 0.9761 & 0.5899 & 0.5896 \\
\hline 9 & 0.9899 & 0.9899 & 0.9750 & 0.9748 \\
\hline 10 & 0.4576 & 0.4574 & 0.1698 & 0.1696 \\
\hline 11 & 0.8990 & 0.8988 & 0.3495 & 0.3494 \\
\hline 12 & 0.8319 & 0.8317 & 0.5205 & 0.5202 \\
\hline
\end{tabular}

Table 7

Comparison of MAC of Configuration B using real and complex modes.

\begin{tabular}{rllll}
\hline \multirow{2}{*}{ Model number } & \multicolumn{2}{l}{ MAC 1 } & & MAC 2 \\
\cline { 2 - 5 } & Real modes & Complex modes & & Real modes \\
\hline 1 & 0.9999 & 0.9999 & 0.9993 & 0.9993 \\
2 & 0.9986 & 0.9986 & 0.9829 & 0.9829 \\
3 & 0.9985 & 0.9985 & 0.9826 & 0.9826 \\
4 & 0.9986 & 0.9986 & 0.9831 & 0.9831 \\
5 & 0.9952 & 0.9951 & 0.9382 & 0.9381 \\
6 & 0.9959 & 0.9959 & 0.9299 & 0.9299 \\
8 & 0.9812 & 0.9811 & 0.8117 & 0.8116 \\
9 & 0.9862 & 0.9861 & 0.7851 & 0.7850 \\
10 & 0.9338 & 0.9337 & 0.3187 & 0.3188 \\
11 & 0.9652 & 0.9652 & 0.5451 & 0.5449 \\
& 0.8452 & 0.8451 & 0.4122 & 0.4122 \\
\end{tabular}

where $\mathrm{LS}_{\mathrm{Nom}}$ is the nominal loss factor of the target mode and $L S_{\mathrm{FEM}}$ is the corresponding loss factor obtained from the erroneous FEM.

The variation of loss factor errors for both the target modes (Loss factor 1 and Loss factor 2, respectively) of the sandwich plate with the MAC values of the corresponding modes are shown in Fig. 6 and the corresponding plots for Configuration B are shown in Fig. 7. It should be noted that generally, the model with MAC values above 0.9 are considered as a successful correlation and the model is assumed to be fit for further analysis. From Fig. 6, it can be seen that a negligible change in the MAC value (0.005) may result in the abrupt change (as high as 40 percent) in the loss factor. It can also be seen that occasionally the model with a lower MAC is able to give a better prediction of the loss factor than the model having higher MAC. This can also be observed in the honeycomb sandwich panel (Fig. 7). Both the figures indicate that, even if the FEM 


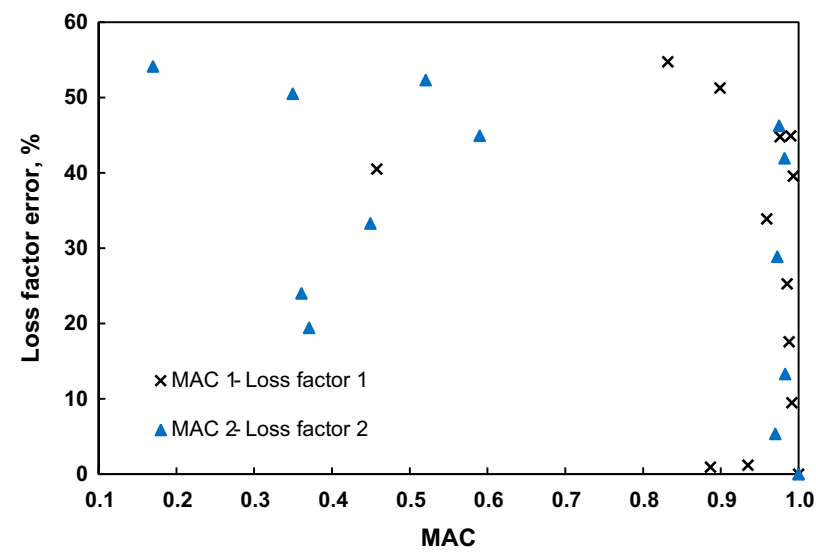

Fig. 6. Variation of MAC of Configuration A with loss factor error.

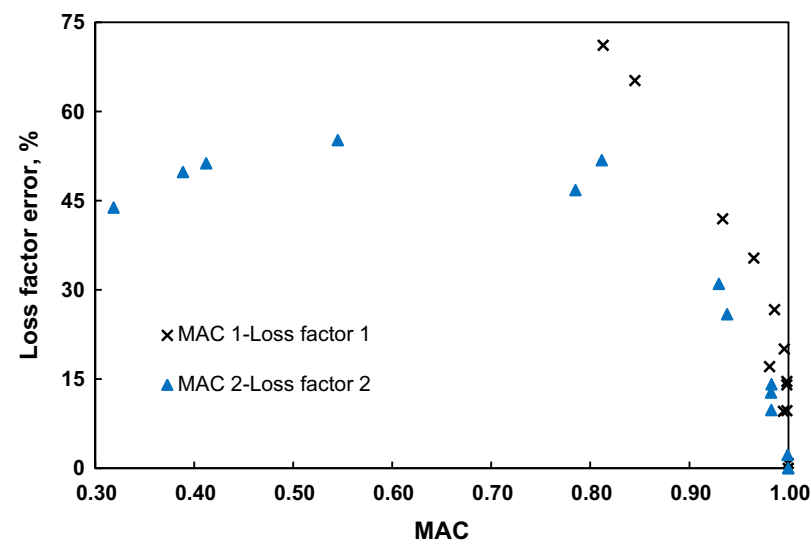

Fig. 7. Variation of MAC of Configuration B with loss factor error.

pass the MAC, the loss factor error can be more than 40 percent, hence it can be concluded that the MAC fails to correlate the modal loss factor of the viscoelastic system. This is primarily due to the definition of MAC, which is a vector correlation between the two modes. If the two mode shapes are identical or its shapes are matched well, then it leads to a MAC correlation value close to one. The results show that, even with similar modes, the energy dissipated in the viscoelastic core can change considerably and hence the error in the prediction of loss factor can be increased. It should also be noted that MAC values will not be altered with the scaling of the modes, but the strain energy and hence the loss factor will differ for that mode.

The NCO diagonal values of the two target modes and the corresponding loss factor errors for Configuration A are shown in Fig. 8 and those for Configuration B are shown in Fig. 9. The off-diagonal terms in the NCO matrix for all the models whose diagonal terms are above 0.9 are all less than 0.1 . Hence, as per the conventional correlation criteria, all these models pass the NCO check. In Fig. 8, many models with NCO values very close to 1 (0.99 or above) also give errors in the loss factor as high as 54 percent. It can also be observed that some models fail the NCO check but are able to give a better prediction of the loss factor than one which passes the NCO check. From Fig. 9, it can be observed that models which pass the NCO criteria can incorrectly estimate the loss factor, in this case with as high as 71 percent error. Similar to MAC, the NCO check also fails to indicate the loss factor of the viscoelastic systems. It is worth mentioning that the MAC and NCO values calculated using all the tri-axial modes (28 tri-axial accelerometers) having 84 dofs for each target modes also gave the same MAC and NCO values (up to the third decimal place) as the target modes of the systems considered here have a predominant motion only in the $Z$ direction.

\subsubsection{Base force assurance criterion and the loss factor}

It was shown in Ref. [27] that BFAC can correlate the forced dynamic characteristics of lightly damped (less than 2 percent critical damping) spacecraft under base excitation. To calculate the BFAC, the base force under a harmonic excitation of $1 \mathrm{~g}$ (where $\mathrm{g}$ is the acceleration due to gravity, $9.81 \mathrm{~m} \mathrm{~s}^{-2}$ ) in the $Z$ direction at the base node for all the models has been determined using a MATLAB [36] program. Then BFAC is calculated using the equation [27]

$$
\text { BFAC }_{j k}=\frac{\left(\mathbf{P}_{j}^{\mathrm{T}} \mathbf{F}_{k}\right)^{2}}{\left(\mathbf{P}_{j}^{\mathrm{T}} \mathbf{P}_{j}\right)\left(\mathbf{F}_{k}^{\mathrm{T}} \mathbf{F}_{k}\right)} .
$$




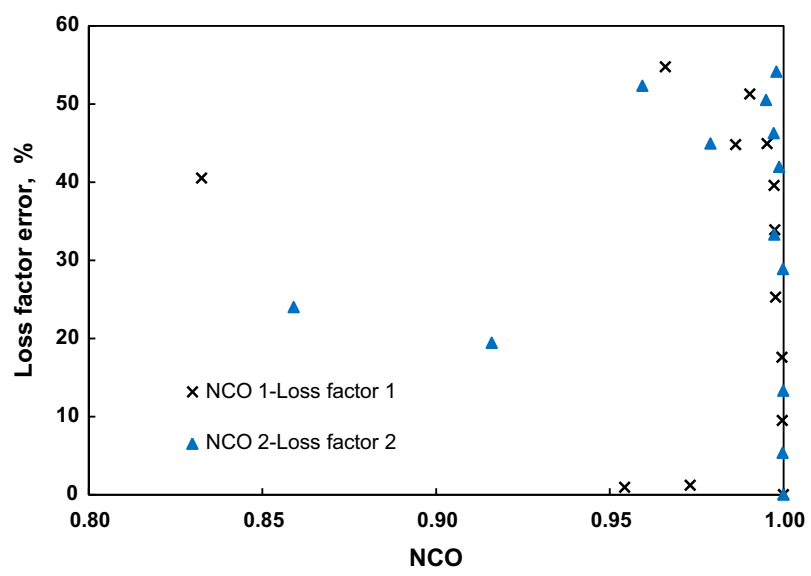

Fig. 8. Variation of NCO of Configuration A with loss factor error.

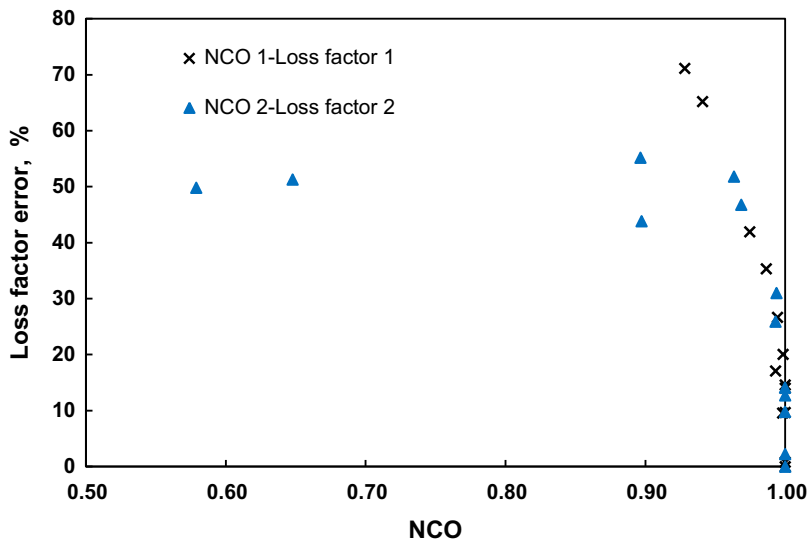

Fig. 9. Variation of NCO of Configuration B with loss factor error.

where $\mathbf{P}$ is the absolute value of the transmitted forces to the base obtained using the nominal FEM and $\mathbf{F}$ is the corresponding absolute value obtained from the erroneous FEMs and the subscripts $j$ and $k$ vary from 1 to 6 for a statically determinate structure. Here, it should be noted that both $\mathbf{P}$ and $\mathbf{F}$ are functions of frequency and each of these parameters contains six columns; the first three are the forces in each of the axes and the last three columns correspond to the moments. Therefore, BFAC is a square matrix of size 6 for a single point constrained system or a system with interfaces are rigidly connected to a single location as in this study and its values vary from 0.0 to 1.0. The first three diagonal values in this matrix correspond to the correlation of forces in three directions and the last three diagonal values correspond to the moment correlations. The BFAC values for both the sandwich plate and the honeycomb sandwich panel were then computed using the forces in the range of $0.0-100.0 \mathrm{~Hz}$ for sandwich plate and $0.0-200.0 \mathrm{~Hz}$ for the sandwich panel. As there are two target modes in the frequency band of interest, the average loss factor of these two target modes has been used for this computation. The BFAC value for the forces in the $Z$ direction with average loss factor errors are shown in Fig. 10. It has been observed that, BFAC in the direction of excitation ( $Z$ direction) gives good correlation with the average loss factor errors for both the systems. It can also be seen that a definite value of BFAC indicates the possible error in the average loss factor. If the measured base force is available (can be determined during the base excitation of the system using a force measuring device), then it can be compared with the analytical counterpart using Eq. (25).

It is worth mentioning that the BFAC also give a very good correlation with the acceleration response error. For example, the absolute response at five different locations on the sandwich panel (shown in Fig. 2) in the $0.0-200.0 \mathrm{~Hz}$ range were calculated under $1 g$ excitation in the $Z$ direction and its error with the peak nominal parameters was determined. The variation of BFAC with average acceleration response error computed using five different locations for this system is shown in Fig. 11 and it can be observed that there is a reasonable correlation between these two parameters.

\subsubsection{The effect of temperature on the standard correlation methods}

As the viscoelastic properties change with temperature and frequency, the properties at different temperatures were used to create different FEMs of the sandwich plate and the honeycomb sandwich panel to assess the effect of temperature 
(a)

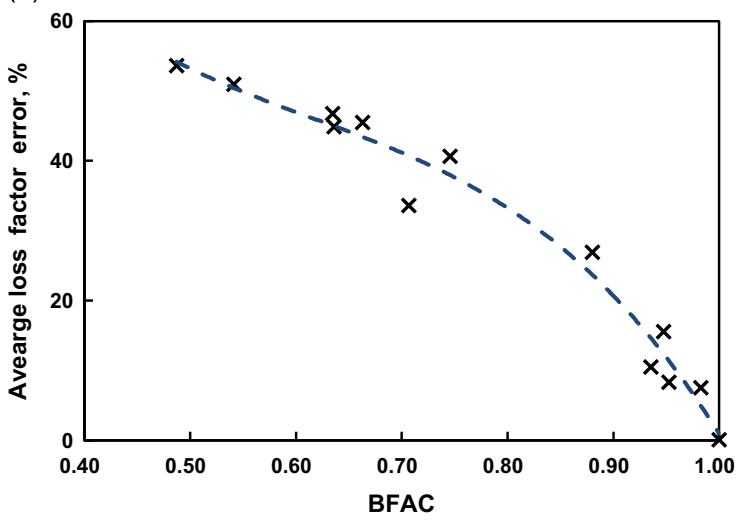

(b)

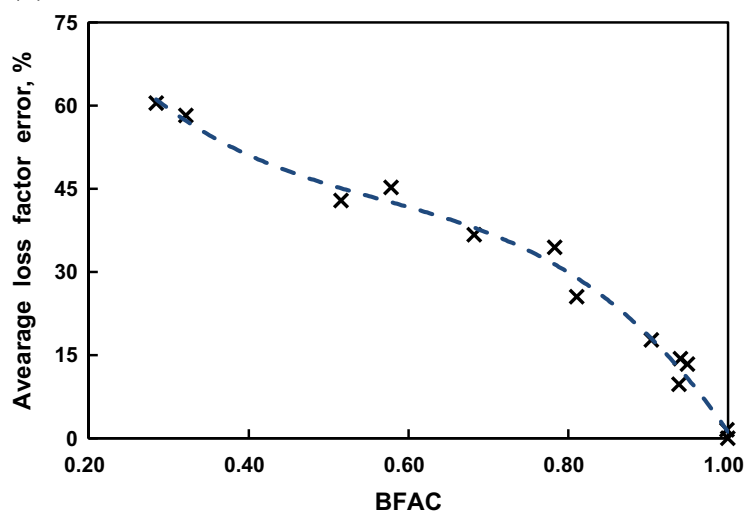

Fig. 10. Variation of BFAC of with average loss factor error: (a) Configuration A and (b) Configuration B.

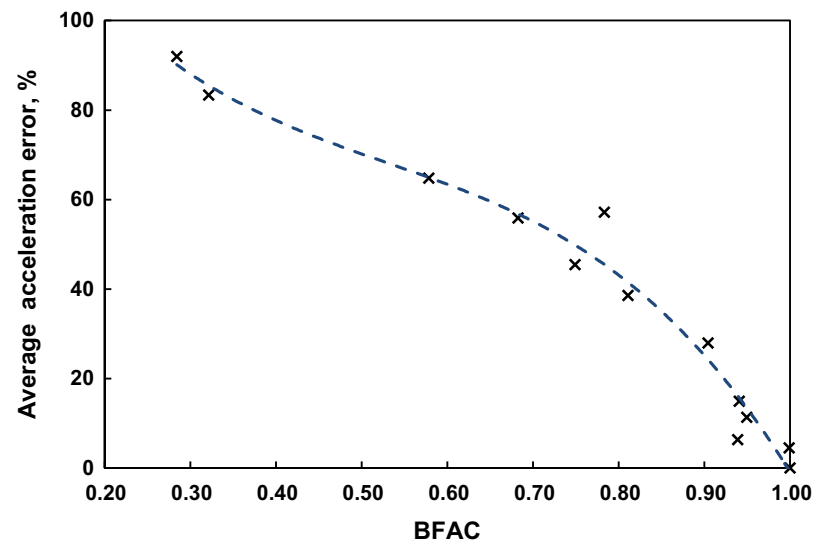

Fig. 11. Variation of BFAC of Configuration B with the average acceleration error.

on the MAC and the NCO check. It is assumed that the variation of viscoelastic properties over the range of frequencies is negligible and the property at $50 \mathrm{~Hz}$ is taken at $10-70{ }^{\circ} \mathrm{C}$ to create different models of the sandwich plate and the property at $132 \mathrm{~Hz}$ is taken at $10-70{ }^{\circ} \mathrm{C}$ for the honeycomb sandwich panel. The approximate frequency at which the viscoelastic properties are chosen for both the systems is based on the average frequency of the two target modes shown in Table 3 . The analysis also neglected the thermal deformation. The viscoelastic properties at $30{ }^{\circ} \mathrm{C}$ were considered for the nominal or base line model as in the earlier case. The required shear modulus of the viscoelastic core was estimated from the catalog [28] at the required temperatures and it is shown in Table 8. The density and the Poisson's ratio of the core were kept the same as the nominal model. The effect of temperature on the material properties of the face sheets is significantly lower than the viscoelastic core in the temperature range used in the analysis and hence neglected.

Another set of models was created using the nominal FEM of the aluminium sandwich plate by modifying the properties of 50 percent of the viscoelastic elements with material properties at $10-70{ }^{\circ} \mathrm{C}$. All of these elements are selected in such a way that it splits the plate into two halves along the length (X-axis in Fig. 1$)$. The models therefore simulate that only half of

Table 8

Viscoelastic properties at different temperatures.

\begin{tabular}{lll}
\hline \multirow{2}{*}{ Temperature $\left({ }^{\circ} \mathrm{C}\right)$} & \multicolumn{2}{l}{ Shear modulus, $G(\mathrm{MPa})$} \\
\cline { 2 - 3 } & Frequency $=50 \mathrm{~Hz}$ & Frequency $=132 \mathrm{~Hz}$ \\
\hline 10 & 10.0 & 15.0 \\
20 & 4.5 & 6.5 \\
40 & 1.0 & 1.6 \\
50 & 0.45 & 0.7 \\
60 & 0.4 & 0.55 \\
70 & 0.3 & 0.4 \\
\hline
\end{tabular}


the structure is under the temperature effect. Then the MAC and the NCO values for both the target modes were calculated using these models, which correspond to different temperatures, and the variation of the MAC and the NCO values with temperature are shown in Figs. 12 and 13, respectively. It can be seen that practically there is no difference in the NCO values calculated at the different temperatures and there is a maximum of 2 percent change in the MAC value. Although the viscoelastic property was highly different for $10^{\circ} \mathrm{C}$, this change is not observed in the MAC or NCO values for this model. This is due to the minor changes in the mode shapes of the models considered. Since only material properties were changed, the difference in the modes from the nominal mode is minimal in this simple structure. Similar results (a maximum of 2 percent change in the MAC and negligible change in the NCO values) were obtained for a comparable sandwich plate which has a viscoelastic core material only in 50 percent of the length (remaining core filled with aluminium) and the viscoelastic material was affected with temperature variation from 10 to $70{ }^{\circ} \mathrm{C}$.

Using the nominal FEM of Configuration B, six other FEMs were created by considering the variation of viscoelastic properties with temperature from 10 to $70{ }^{\circ} \mathrm{C}$. The temperature-dependent material properties shown in Table 8 were used to create different models. The MAC and NCO values of the two target modes were calculated using these models and the variation of MAC with the temperature is shown in Fig. 14. The MAC of the second target mode varies from 1.0 to 0.64 and the lowest MAC value occurs at $70{ }^{\circ} \mathrm{C}$. As in the sandwich plate models, the NCO values of both the target modes did not vary with temperature and hence are not shown here. The mass weighting matrix in the NCO correlation nullifies the changes in the mode shape, and hence the changes in the NCO are minimal. It can be concluded that there is no necessity to compute the NCO values for different temperatures for these types of systems, if the uncertainty exists only in the material properties.

The modal loss factor sensitivity with the temperature is also performed for Configuration A. To calculate the modal loss factor using Eq. (1), the temperature and frequency-dependent material loss factor is used. The material loss factor remains constant $\left(\eta_{v}=1\right)$ over the temperature range from 30 to $60{ }^{\circ} \mathrm{C}$ whereas it is taken as 0.9 for $20{ }^{\circ} \mathrm{C}$ and $70{ }^{\circ} \mathrm{C}$ and 0.55 for the $10{ }^{\circ} \mathrm{C}$ at $50 \mathrm{~Hz}$ [28]. Using these parameters, the modal loss factor of the two target modes for Configuration A is shown in Table 9. It can be observed that the smallest loss factor occurs at $10{ }^{\circ} \mathrm{C}$ whereas the highest loss factor can be obtained at $40{ }^{\circ} \mathrm{C}$.

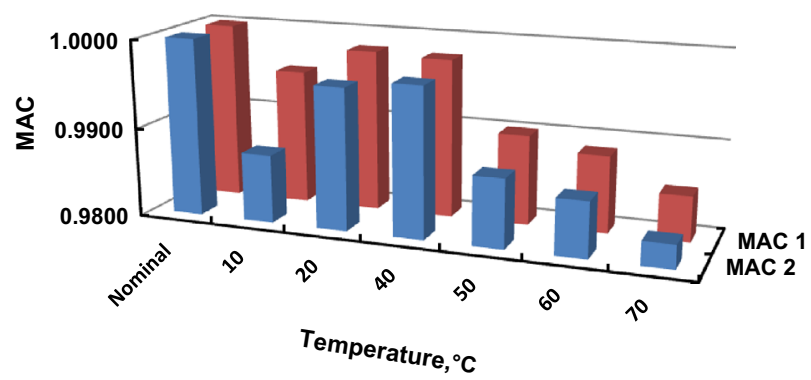

Fig. 12. MAC at different temperatures for Configuration A.

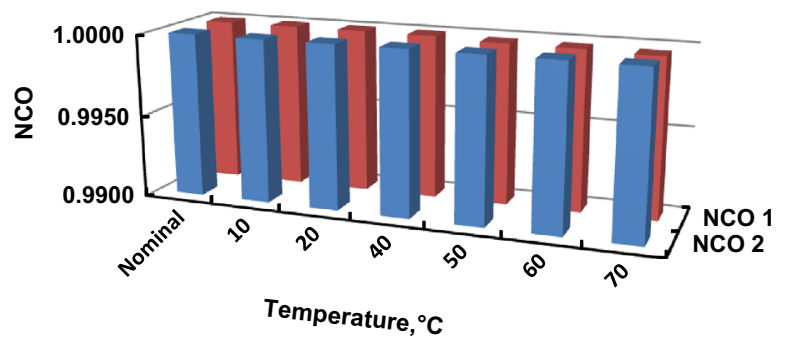

Fig. 13. NCO at different temperatures for Configuration A.

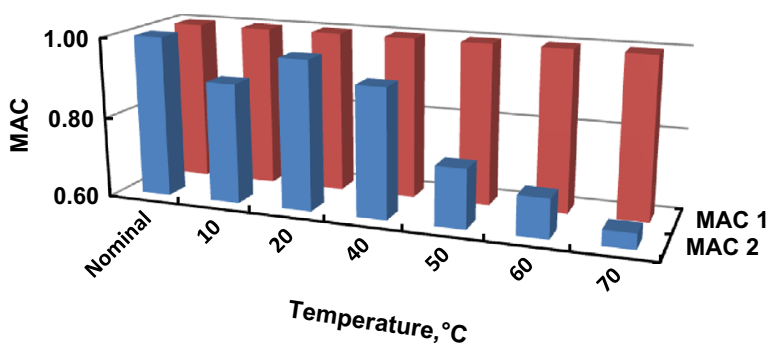

Fig. 14. MAC at different temperatures for Configuration B. 
Table 9

Variation of modal loss factor with temperature.

\begin{tabular}{lll}
\hline \multirow{2}{*}{ Temperature $\left({ }^{\circ} \mathrm{C}\right)$} & \multicolumn{2}{l}{ Modal loss factor } \\
\cline { 2 - 3 } & Target mode 1 & Target mode 2 \\
\hline 30 (Nominal) & 0.1496 & 0.1271 \\
10 & 0.0296 & 0.0405 \\
20 & 0.0872 & 0.0982 \\
40 & 0.1779 & 0.1176 \\
50 & 0.1651 & 0.0878 \\
60 & 0.1593 & 0.0828 \\
70 & 0.1282 & 0.0635 \\
\hline
\end{tabular}

\subsection{Piezoelectric system}

\subsubsection{Dynamic response}

The piezoelectric system shown previously in Fig. 4 has been analyzed under the $0.1 \mathrm{~g}$ base excitation in the $Z$ direction without any electrical circuits and the bottom electrode of the piezoelectric patch, which interfaces with the plate, in a grounded condition. The critical damping ratio was taken as $7.8 \times 10^{-4}[14]$ and the absolute acceleration response at the tip of the cantilever during the third bending mode is shown in Fig. 15. This response has peak amplitude of $27.43 g$ and occurs at $724.6 \mathrm{~Hz}$. Then the piezoelectric electrodes are attached with a resistance $(1 \Omega)$ and an inductor $(0.65 \mathrm{H})$. The dynamic response at the tip with these electrical components is also shown in Fig. 15. The first peak in this figure is the electrical resonance that occurs at $639.7 \mathrm{~Hz}$. These typical values of the electrical parameters are chosen to show a distinct electrical resonance mode. It can be observed that there is a slight decrease in the tip acceleration (7.6 percent) during the third bending mode compared to the system which does not have any electrical circuits. If further reduction in the peak acceleration response is required, the electrical circuit parameters can be tuned. One such option with $R=1000 \Omega$ and $L=0.65 \mathrm{H}$ gives a tip acceleration as low as $4.4 \mathrm{~g}$ during the third bending mode for the same base excitation and this response plot is also shown in Fig. 15.

\subsubsection{Correlation of finite element model of a piezoelectric system}

To understand how the FEM of a piezoelectric system performs under dynamic loading when it possesses a certain value of the MAC, a study has been carried out using the different FEMs generated from the nominal FEM. It is understood that the MAC is not a criterion for the response analysis but it is the most popular correlation method. The FEM generated using the material property shown in Table 2 is considered as the nominal model and the obtained results were taken as the experimental or 'true' results for the correlation study. It is understood that there is a good amount of uncertainty in the properties of piezoelectric material $[13,14]$, and hence ten different intentionally erroneous FEMs were generated from the nominal FEM by varying all the material properties of the system from 0.5 to 25.0 percent of the nominal value shown in Table 2. The percentage increase in the material properties for different erroneous models was chosen as $0.5,0.75,1.0,2.5$, 5.0, 7.5, 10.0, 15.0, 20.0, and 25.0. Geometry, boundary condition and the electrical shunt circuit $(R=1 \Omega, L=0.65 \mathrm{H})$ were kept constant throughout.

Tip acceleration, tip displacement and the peak current in the electrical circuit were computed in the frequency range of $5.0-750.0 \mathrm{~Hz}$ during the base excitation of the system. As in the previous case, the input acceleration was $0.1 \mathrm{~g}$ in the $Z$ direction. The percentage error in the absolute acceleration, the absolute tip displacement for the third bending mode and

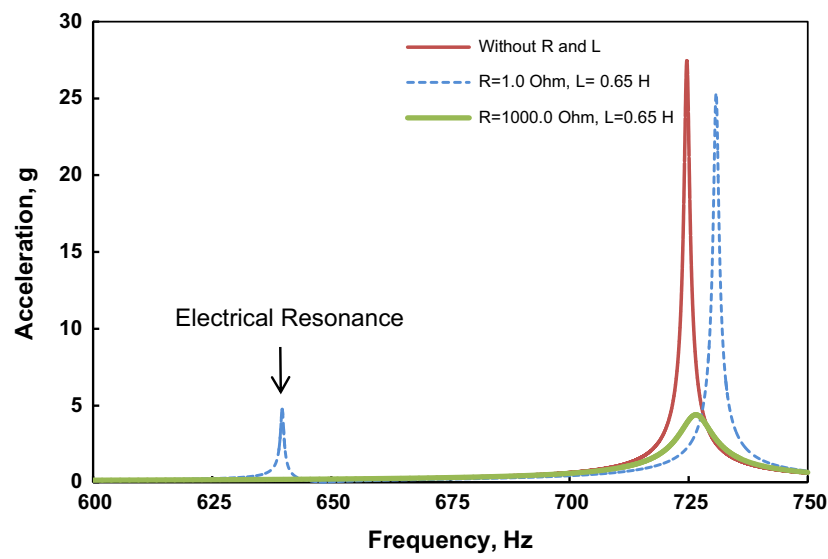

Fig. 15. Tip acceleration with different configurations the of electrical circuit. 
the peak current error in the shunt circuit were also computed. For the computation of the MAC, the third bending mode has been considered and the variation of tip acceleration error with the MAC is shown in Fig. 16. It has been observed that a model with a MAC value very close to 1 (0.998) also gives a high error (say 20 percent) in the acceleration response. In addition to that, models with negligible difference (0.001) in MAC values may also give different levels of acceleration errors. Here, the change in the mode shape is minimal (hence MAC close to 1) because the variations in the FEMs are only in the material properties. However, variations in the material properties change the frequency of vibration and the effective mass of the mode and hence the response. Although Fig. 16a shows some correlation between the MAC and the tip acceleration, it should be noted that practically it will be very difficult to determine the very small changes (in the order of $10^{-3}$ ) in the MAC values. Thus, effectively there is no correlation between the MAC and the model capability to predict the acceleration response. It should also be noted that generally a model with the MAC $>0.9$ is considered to be a successful model [25,26], but here it fails to represent the acceleration response within the limit (e.g., $<2$ percent error).

The variation of tip displacement error with MAC of the third bending mode also shows a similar trend to the acceleration and hence is not shown here. Fig. 16b shows the variation of peak current error with the MAC. As in the case of acceleration error, neither the dynamic displacement nor the electric current shows any useful correlation with the MAC value. Also for the similar MAC values (maximum difference in the order of $10^{-3}$ ), error in current may drastically change. Hence, MAC is not a good correlation tool to assess the FEM capability to predict the dynamic characteristics of the multiphysics system. The MAC values that are very close to 1 are primarily due to the nature of the models considered in this study, which are generated by changing the material properties and the structure is a simple cantilever. Hence, the change in the mode shape is negligible compared to the nominal mode. However, the purpose here is to show that there are FEMs, which can easily pass the MAC, but fail in the prediction of dynamic characteristics of the multi-physics system.

\subsubsection{Current assurance criterion}

It has been shown in the previous section that the conventional correlation method, the MAC, fails to correlate the dynamic characteristics of the multi-physics system, as it is a pure vector correlation of two deformed shapes. The variation

(a)

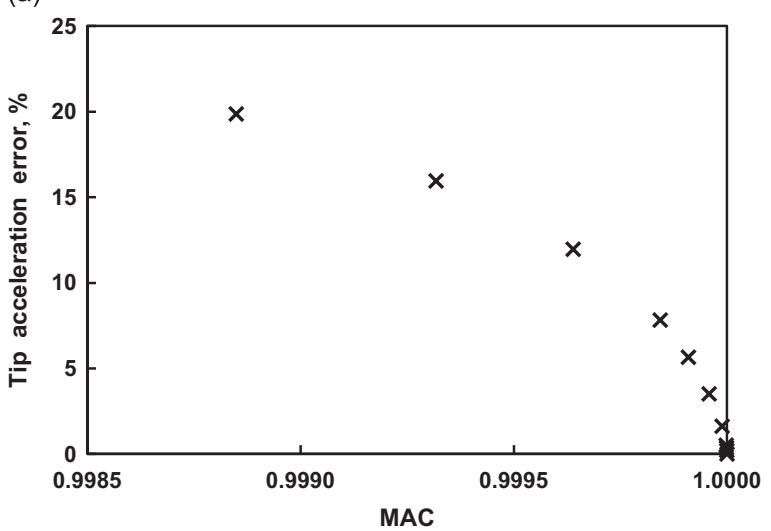

(b)

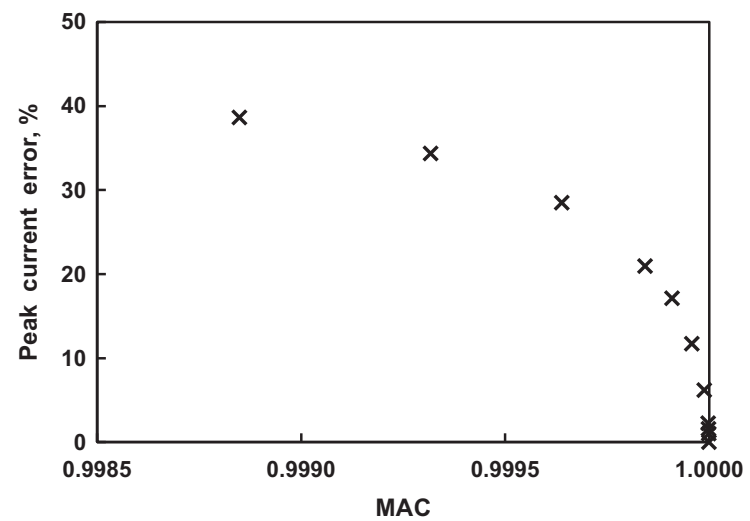

Fig. 16. Variation of tip acceleration error with the MAC for Configuration C: (a) acceleration and (b) current.

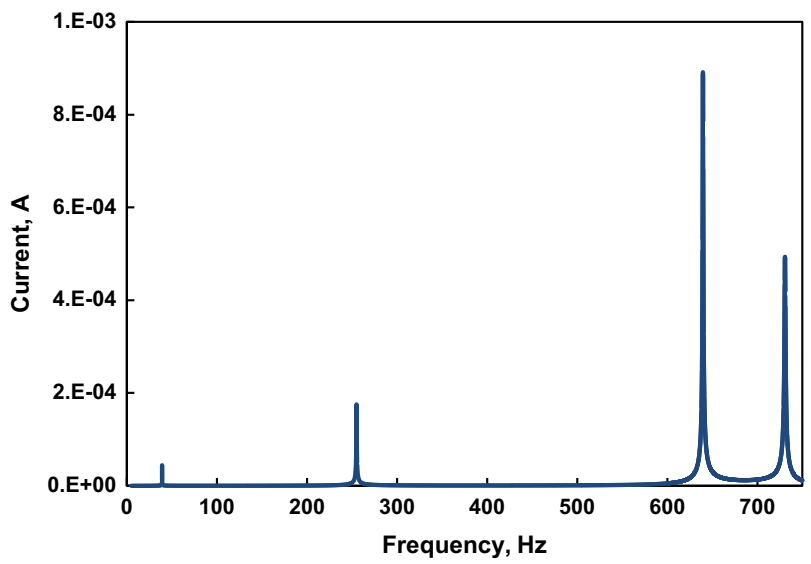

Fig. 17. Variation of current with frequency $(R=1 \Omega, L=0.65 \mathrm{H})$. 
of the nominal value of the current with frequency, when the piezoelectric structure is connected with the electric circuit is excited with a harmonic input $(0.1 \mathrm{~g})$ at the base is shown in Fig. 17. The maximum current in the circuit occurs during the electrical resonance and in this chosen configuration it occurs at $639.7 \mathrm{~Hz}$. The other three peaks in the figure correspond to the first three bending modes of the structure, which occur at $39.4 \mathrm{~Hz}, 255.1 \mathrm{~Hz}$, and $730.8 \mathrm{~Hz}$, respectively. It can be noted that, unlike acceleration or displacement, current in the shunt circuit does not depend on the location and will be unique for each circuit. However, current in the circuit varies with the frequency and the current values in the frequency range contain important information about the dynamics of the system. Using this property, in line with the base force assurance criterion, a current assurance criterion is defined as

$$
\mathrm{CAC}=\frac{\left(I_{\mathrm{Exp}}^{\mathrm{T}} I_{\mathrm{FEM}}\right)^{2}}{\left(I_{\mathrm{Exp}}^{\mathrm{T}} I_{\mathrm{Exp}}\right)\left(I_{\mathrm{FEM}}^{\mathrm{T}} I_{\mathrm{FEM}}\right)}
$$

where $I_{\mathrm{Exp}}$ and $I_{\mathrm{FEM}}$ are the experimental and the analytically calculated absolute current values in the electrical circuit. It should be noted that both of these quantities are functions of frequency and all the values of current in the frequency domain of interest need to be included for the CAC computation. This quality indicator varies from 0.0 to 1.0 where 1.0 indicates a perfect correlation between the experimental and the analytical result. If there are a number of piezoelectric patches connected with separate electrical networks or there are a series of shunt circuits, then for such different current values, separate CAC need to be evaluated for the purpose of correlation. During dynamic testing, the value of the current can be measured for the frequency range of interest.

As in the earlier case, the current obtained from the nominal FEM is taken as the experimental value and those obtained from the different erroneous FEM generated by varying the material properties were taken as the analytical values. The CAC for the different FEMs were calculated in the frequency band of 5.0-750.0 Hz and the variation of CAC with the absolute tip acceleration error for those models is shown in Fig. 18a. It can be seen that, for a specific value of the CAC, the error in the acceleration response is unique. Hence, the CAC can be used as a quality indicator for the acceleration response of this type of system.

The variation of tip dynamic displacement error with the CAC shown in Fig. 18b and Fig. 18c shows the CAC variation with the error in the peak current in the circuit; both these quantities correlate well with the CAC. For the numerical example analyzed here, a CAC value greater than 0.2 always assures that the error in the acceleration, dynamic displacement and the peak current is within 2 percent, being an acceptable value for the most practical problems.

(a)

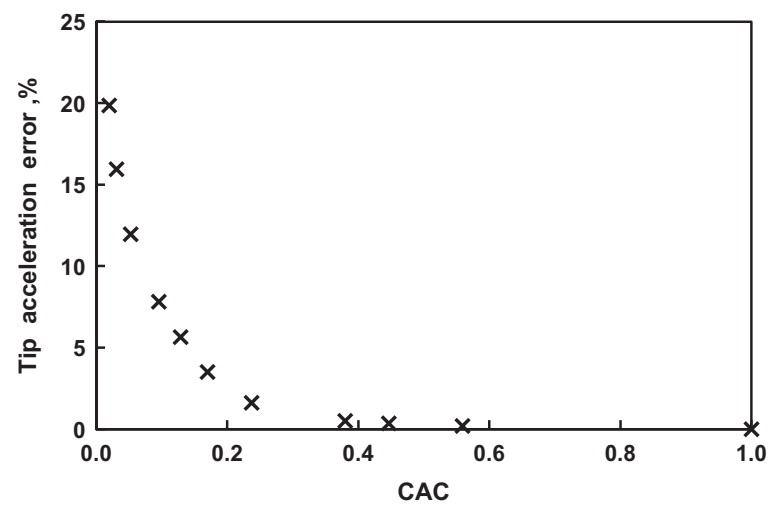

(b)

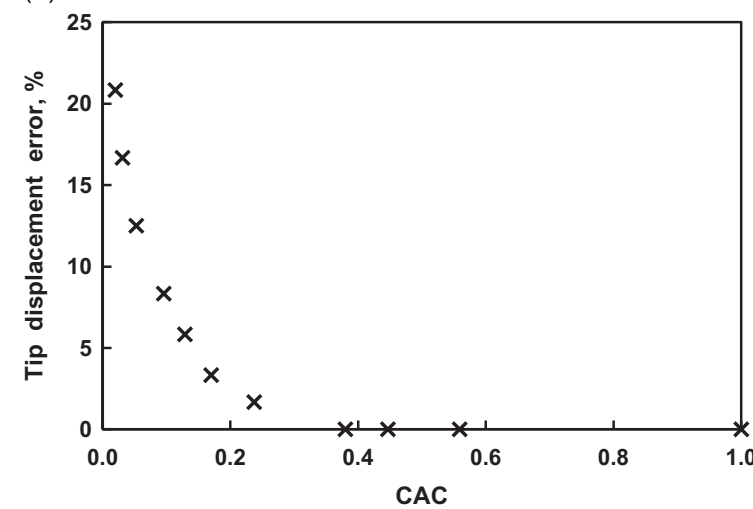

(c)

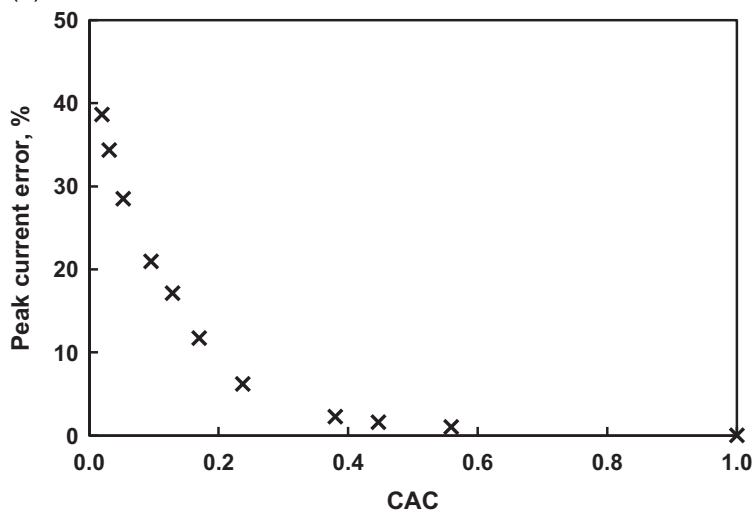

Fig. 18. Variation of dynamic characteristics error with the CAC: (a) acceleration, (b) displacement and (c) current. 


\section{Conclusion}

Viscoelastic materials and electric circuit-fed piezoelectric systems have been used for vibration reduction and the correlation of FEMs of such systems has been studied in this work. The effectiveness of the MAC and NCO check on the prediction of the modal loss factor for two subsystems has been analyzed using intentionally erroneous FEMs. To assess the effect of damping on the MAC, the MAC was computed using the complex modes and it was observed that there is no significant change compared to the corresponding value determined from the real modes. Hence, only real modes were used for further analysis and observed that these correlation methods do not give any indication on the capability of the model to predict the loss factor. The recently introduced BFAC is found to be more effective than the MAC or NCO check in the prediction of loss factor of such systems. Also, the effect of temperature on the MAC and NCO check were studied and noted that there is a change in the MAC values with temperature. However, the NCO values do not vary within the temperature band considered $\left(10-70^{\circ} \mathrm{C}\right)$ for both systems. This indicates that the FEM does not need to be correlated at different temperatures using the NCO criterion if the uncertainty is mainly attributed to the material properties of the systems considered.

In addition, the FEM of a structure attached with an electric circuit-fed piezoelectric material has been reviewed. This multi-physics system consists of a cantilever plate, a piezoelectric patch on the top of the plate and a RL circuit in series. The FEM correlation of the coupled system is carried out using the nominal FEM and the intentionally erroneous models and observed that MAC does not display any useful correlation to the dynamic characteristics. This is because even if there is no observable change in the mode shapes as in this cantilever structure, the response of the structure still varies. However, as the MAC is based on mode shape correlation it will still indicate a high value. A new correlation tool identified as the CAC is introduced by comparing the electric current in the circuit during the harmonic excitation obtained from the nominal model, and that noted from the erroneous FEM. A good correlation has been obtained between the CAC and the structural dynamic characteristics error and the error in the peak current. For the typical problem considered in this study, a CAC value of 0.2 always assures that the error in the response is within 2 percent, hence the CAC can be used for the assessment of FEMs of these types of multi-physics systems.

\section{Acknowledgments}

The authors wish to acknowledge the Commonwealth Scholarship Commission, UK (No. INCS-2010-188), for their funding. They would also like to thank Thomas K. Joseph, Scientist - ISRO Satellite Centre, Bangalore for the help in Nastran software.

\section{Appendix A. An approximate expression for the modal loss factor}

The modal loss factor of the complex structure with viscoelastic damping was calculated using the approximate method proposed by Johnson and Kienholz [11]. This approach is based on the modal strain energy method introduced by Ungar [9] and the derivation for the modal loss factor using this method is given below [11].

The equation for the free vibration of viscoelastic system can be written as

$$
\mathbf{M} \ddot{\mathbf{x}}+\left(\mathbf{K}_{\mathrm{Re}}+\mathbf{i} \mathbf{K}_{\mathrm{Im}}\right) \mathbf{x}=0
$$

$>$ where $\mathbf{K}_{\mathrm{Re}}$ and $\mathbf{K}_{\mathrm{Im}}$ are the real and the imaginary part of the stiffness matrix. The equation can be converted to an eigenvalue problem by considering a solution,

$$
\mathbf{X}=\overline{\boldsymbol{\phi}}^{r} \mathrm{e}^{\mathrm{i} \overline{\mathrm{\lambda}}_{r} t}
$$

where $\bar{\lambda}_{r}$ and $\bar{\phi}$ are the $r$ th complex eigenvalue and eigenvector. The complex eigenvalue can also be written as

$$
\bar{\lambda}_{r}=\lambda_{r} \sqrt{1+\mathrm{i} \eta^{r}}
$$

where $\eta^{\mathrm{r}}$ represents the loss factor of the $r$ th mode. For the complex stiffness matrix considered in Eq. (A.1), the Raleigh's quotient formula can be written after dropping the mode index $r$ as

$$
\bar{\lambda}^{2}=\lambda^{2}+\mathrm{i} \eta \lambda^{2}=\frac{\overline{\boldsymbol{\phi}}^{\mathrm{T}} \mathbf{K}_{\mathrm{Re}} \overline{\boldsymbol{\phi}}}{\overline{\boldsymbol{\Phi}}^{\mathrm{T}} \mathbf{M} \overline{\boldsymbol{\phi}}}+\mathrm{i} \frac{\overline{\boldsymbol{\phi}}^{\mathrm{T}} \mathbf{K}_{\mathrm{Im}} \overline{\boldsymbol{\phi}}}{\overline{\boldsymbol{\phi}}^{\mathrm{T}} \mathbf{M} \overline{\boldsymbol{\phi}}} .
$$

The loss factor can be approximated by replacing the complex eigenvector $\bar{\phi}$ with the real eigenvector, $\phi$ in Eq. (A.4). In this case, the real eigenvector can be obtained by neglecting the imaginary part of the stiffness matrix and solving the pure 
elastic eigenvalue problem. Then, equating the real and imaginary parts gives

$$
\begin{gathered}
\lambda^{2}=\frac{\boldsymbol{\phi}^{\mathrm{T}} \mathbf{K}_{\mathrm{Re}} \boldsymbol{\phi}}{\boldsymbol{\phi}^{\mathrm{T}} M \boldsymbol{\phi}} \\
\eta \lambda^{2}=\frac{\boldsymbol{\phi}^{\mathrm{T}} \mathbf{K}_{\mathrm{Im}} \boldsymbol{\phi}}{\boldsymbol{\phi}^{\mathrm{T}} M \boldsymbol{\phi}} .
\end{gathered}
$$

The stiffness matrix obtained using the finite element analysis can be segregated to the terms corresponding to the elastic elements, $\mathbf{K}_{e}$ and the viscoelastic elements, $\mathbf{K}_{v}$. The second matrix will be a complex matrix, but for a single viscoelastic material, its imaginary part $\left(\mathbf{K}_{v I}\right)$ and real part $\left(\mathbf{K}_{v R}\right)$ follow the relation:

$$
\mathbf{K}_{v I}=\eta_{v} \mathbf{K}_{v R}
$$

where $\eta_{v}$ is the loss factor of the viscoelastic material at the $r$ th resonant frequency. Hence,

$$
\mathbf{K}_{v}=\mathbf{K}_{v R}+\mathrm{i} \mathbf{K}_{v I}=\mathbf{K}_{v R}\left(1+\mathrm{i} \eta_{v}\right) \text {. }
$$

Assuming that only the complex part of $\mathbf{K}_{v}$ contributes to the imaginary part of the system stiffness matrix, then

$$
\mathbf{K}_{\mathrm{Im}}=\mathbf{K}_{v I} \text {. }
$$

Let $V_{T}$ be the total strain energy obtained when a purely elastic normal mode analysis is performed. This can be calculated using the normal mode as

$$
V_{T}=\boldsymbol{\phi}^{\mathrm{T}} \mathbf{K}_{\mathrm{Re}} \boldsymbol{\phi} .
$$

A portion of the total strain energy will be contributed by the viscoelastic elements and it is given by

$$
V_{v}=\boldsymbol{\phi}^{\mathrm{T}} \mathbf{K}_{v R} \boldsymbol{\phi} .
$$

Using Eqs. (A.5) and (A.6), the modal loss factor can be obtained as

$$
\eta=\frac{\phi^{\mathrm{T}} \mathbf{K}_{\mathrm{Im}} \boldsymbol{\phi}}{\boldsymbol{\phi}^{\mathrm{T}} \mathbf{K}_{\mathrm{Re}} \boldsymbol{\phi}}
$$

Using the relationship shown in Eqs. (A.9) and (A.7), the above equation can be re-written as

$$
\eta=\frac{\eta_{v} \boldsymbol{\phi}^{\mathrm{T}} \mathbf{K}_{v R} \boldsymbol{\phi}}{\boldsymbol{\phi}^{\mathrm{T}} \mathbf{K}_{\mathrm{Re}} \boldsymbol{\phi}} .
$$

Substituting Eqs. (A.10) and (A.11) in Eq. (A.13) and reintroducing mode superscript $r$ gives the loss factor as

$$
\eta^{r}=\eta_{v}\left(\frac{V_{v}^{r}}{V_{T}^{r}}\right)
$$

\section{Appendix B. Sub-matrices in the electro-elastic equilibrium equations}

Individual terms in the equilibrium equations of the electro-mechanical system (given in Eqs. (4) and (5)) are shown in Table B.1 [17].

Table B.1

Electro-elastic parameters.

\begin{tabular}{ll}
\hline Parameter & Description \\
\hline $\mathbf{M}=\rho \iiint_{V} \mathbf{N}_{x}^{\mathrm{T}} \mathbf{N}_{x} \mathrm{~d} V$ & Mass matrix \\
$\mathbf{K}_{x x}=\iiint_{V} \mathbf{B}_{x}^{\mathrm{T}} \mathbf{C} \mathbf{B}_{x} \mathrm{~d} V$ & Stiffness matrix \\
$\mathbf{K}_{x v}=\iiint_{V} \mathbf{B}_{x}^{\mathrm{T}} \mathbf{P B}_{v} \mathrm{~d} V$ & Piezoelectric stiffness matrix \\
$\mathbf{f}_{B}=\iiint_{V} \mathbf{N}_{x}^{\mathrm{T}} \mathbf{N}_{\bar{f}} \mathrm{~d} V \overline{\mathbf{f}}_{i}$ & Body force \\
$\mathbf{f}_{S}=\iiint_{S_{1}} \mathbf{N}_{x}^{\mathrm{T}} \mathbf{N}_{\bar{\sigma}} \mathrm{d} S \bar{\sigma}_{i}$ & Surface force \\
$\mathbf{f}_{p}=\mathbf{N}_{x}^{\mathrm{T}} \mathbf{p}$ & Concentrated force \\
$\mathbf{K}_{v x}=\iiint_{V} \mathbf{B}_{v}^{\mathrm{T}} \mathbf{P}^{\mathrm{T}} \mathbf{B}_{x} \mathrm{~d} V$ & Piezoelectric stiffness matrix \\
$\mathbf{K}_{v v}=-\iiint_{V} \mathbf{B}_{v}^{\mathrm{T}} \mathbf{D} \mathbf{B}_{v} \mathrm{~d} V$ & Dielectric stiffness matrix \\
$\mathbf{q}_{B}=-\iiint_{V} \mathbf{n}_{v} N_{\bar{q}} \mathrm{~d} V \overline{\mathbf{q}}_{i}$ & Body charge \\
$\mathbf{q}_{s}=-\iint_{S_{2}} \mathbf{n}_{v} N_{\bar{q}} \mathrm{~d} S \overline{\mathbf{q}}_{i}^{\prime}$ & Surface charge \\
$\mathbf{q}_{p}=-\mathbf{n}_{v} Q$ & Point charge \\
\hline
\end{tabular}




\section{References}

[1] R.M. Lin, J. Zhu, Finite element model updating using vibration test data under base excitation, Journal of Sound and Vibration 303 (2007) 596-613, http://dx.doi.org/10.1016/j.jsv.2007.01.029.

[2] R.J. Allemang, D.L. Brown, A correlation coefficient for modal vector analysis, Proceedings of First International Modal Analysis Conference, Society for Experimental Mechanics, Connecticut, USA, 1982, pp. 110-116.

[3] D.J. Ewins, Modal Testing Theory, Practice and Application, Engineering Dynamics Series, 2nd ed. Research Studies Press Ltd., Baldock, England, 2000.

[4] N.A.J. Lieven, D.J. Ewins, Spatial correlation of mode shapes: the coordinate modal assurance criterion (COMAC), Proceedings of Sixth International Modal Analysis Conference, Society for Experimental Mechanics, Connecticut, USA, 1988, pp. 690-695.

[5] D. Nefske, S. Sung, Correlation of a coarse mesh finite element model using structural system identification and a frequency response criterion, Proceedings of 14th International Model Analysis Conference, Society for Experimental Mechanics, Connecticut, USA, 1996, pp. 597-602.

[6] R.A.S. Moreira, J.D. Rodrigues, Multilayer damping treatments: modeling and experimental assessment, Journal of Sandwich Structures and Materials 12 (2010) 181-198, http://dx.doi.org/10.1177/1099636209104530.

[7] M.D. Rao, Recent applications of viscoelastic damping for noise control in automobiles and commercial airplanes, Journal of Sound and Vibration 262 (2003) 457-474, http://dx.doi.org/10.1016/s0022-460x(03)00106-8.

[8] D.J. Mead, Passive Vibration Control, John Wiley \& Sons Ltd., West Sussex, England, 2000.

[9] E.E. Ungar, E.M. Kerwin, Loss factors of viscoelastic systems in terms of energy concepts, The Journal of the Acoustical Society of America 34 (1962) 954-957.

[10] C.T. Sun, B.V. Sankar, V.S. Rao, Damping and vibration control of unidirectional composite laminates using add-on viscoelastic materials, Journal of Sound and Vibration 139 (1990) 277-287, http://dx.doi.org/10.1016/0022-460x(90)90888-7.

[11] C.D. Johnson, D.A. Kienholz, Finite-element prediction of damping in structures with constrained viscoelastic layers, AIAA Journal 20 (1982) 1284-1290, http://dx.doi.org/10.2514/3.51190.

[12] P. Bangarubabu, K. Kishore Kumar, Y. Krishna, Damping effect of viscoelastic materials on sandwich beams, International Conference on Trends in Industrial and Mechanical Engineering, Planetary Scientific Research Centre, Dubai, 2012.

[13] J. Becker, O. Fein, M. Maess, L. Gaul, Finite element-based analysis of shunted piezoelectric structures for vibration damping, Computers and Structures 84 (2006) 2340-2350, http://dx.doi.org/10.1016/j.compstruc.2006.08.067.

[14] J.B. Min, K.P. Duffy, A.J. Provenza, Shunted piezoelectric vibration damping analysis including centrifugal loading effects, 51st AIAA/ASME/ASCE/AHS/ASC Structures, Structural Dynamics, and Materials Conference, Orlando, Florida, AIAA 2010-2716, 2010.

[15] G. Caruso, A critical analysis of electric shunt circuits employed in piezoelectric passive vibration damping, Smart Materials and Structures 10 (2001) 1059-1068, http://dx.doi.org/10.1088/0964-1726/10/5/322.

[16] E.P. Eernisse, Variational method for electroelastic vibration analysis, IEEE Transactions on Sonics and Ultrasonics 14 (1967) 153-160.

[17] H. Allik, T.J.R. Hughes, Finite element method for piezoelectric vibration, International Journal for Numerical Methods in Engineering 2 (1970) 151-157, http://dx.doi.org/10.1002/nme.1620020202.

[18] P. Cupial, Three-dimensional natural vibration analysis and energy considerations for a piezoelectric rectangular plate, Journal of Sound and Vibration 283 (2005) 1093-1113, http://dx.doi.org/10.1016/j.jsv.2004.06.019.

[19] H.S. Tzou, R. Ye, Piezothermoelasticity and precision control of piezoelectric systems - theory and finite-element analysis, Journal of Vibration and Acoustics-Transactions of the ASME 116 (1994) 489-495, http://dx.doi.org/10.1115/1.2930454.

[20] J.S. Wang, D.F. Ostergaard, A finite element-electric circuit coupled simulation method for piezoelectric transducer, Proceedings of IEEE Ultrasonics Symposium, IEEE, Washington, 1999, pp. 1105-1108.

[21] M.C. Reaves, L.G. Horta, Piezoelectric Actuator Modeling Using Msc/Nastran and Matlab, NASA/TM-2003-212651, Langley Research Center, 2003.

[22] M.W. Al-Hazmi, Finite element analysis of cantilever plate structure excited by patches of piezoelectric actuators, Proceedings of 11th IEEE Intersociety Conference on Thermal and Thermomechanical Phenomena in Electronic Systems 1-3, IEEE, USA, 2008, pp. 809-814.

[23] B. Seba, J. Ni, B. Lohmann, Vibration attenuation using a piezoelectric shunt circuit based on finite element method analysis, Smart Materials and Structures 15 (2006) 509-517, http://dx.doi.org/10.1088/0964-1726/15/2/034

[24] Ansys Multiphysics, 14.0 ed, ANSYS, Inc., Canonsburg, Pennsylvania, 2011.

[25] Loads Analysis of Spacecraft and Payloads, NASA-STD-5002, URL: 〈https://standards.nasa.gov/training/nasa-std-5002/index.html〉 (accessed 23.03.11).

[26] Modal Survey Assessment, ECSS-E-ST-32-11C, European Space Agency, 2008, p. 49.

[27] K.K. Sairajan, G.S. Aglietti, Study of the correlation criteria for base excitation of spacecraft structures, Journal of Spacecraft and Rockets 51 (2014) 106-116, http://dx.doi.org/10.2514/1.A32457.

[28] 3Mтм Ultra-Pure Viscoelastic Damping Polymer, Electronics Markets Materials Division, 3M Center, St. Paul, Minnesota, Mar., 2012. URL: 〈http:// multimedia.3m.com/mws/mediawebserver?mwsId=66666UF6EVsSyXTtnxf2oXF6EVtQEVs6EVs6EVs6E666666-\&fn=62508.PDF (accessed 12.02.13).

[29] W.J. Mccalla, Fundamentals of Computer-Aided Circuit Simulation, Kluwer Academic Publishers, Boston, 1988.

[30] MSC Nastran Users Manual, MSC Software Corporation, California, 2001.

[31] Ansys 14.0 Help, ANSYS, Inc., Canonsburg, Pennsylvania 2011.

[32] Coupled-Field Analysis Guide, ANSYS, Inc., Canonsburg, Pennsylvania 2009.

[33] Y.T. Chung, M.L. Sernaker, Assessment of target mode selection criteria for payload modal survey, Proceedings of 12th International Modal Analysis Conference, Society for Experimental Mechanics, Connecticut, USA, 1994, pp. 272-279.

[34] R. Penrose, A generalised inverse for matrices, Mathematical Proceedings of the Cambridge Philosophical Society 51 (1955) 406-413, http://dx.doi.org/ $10.1017 /$ S0305004100030401

[35] K.K. Sairajan, G.S. Aglietti, Robustness of system equivalent reduction expansion process on spacecraft structure model validation, AIAA Journal 50 (2012) 2376-2388, http://dx.doi.org/10.2514/1.J051476.

[36] Matlab, R2011a ed, MathWorks, Inc., Natick, Massachusetts, 2011. 